UC3M Working papers

Economics

17-03

January , 2017

ISSN 2340-5031
Departamento de Economía

Universidad Carlos III de Madrid

Calle Madrid, 126

28903 Getafe (Spain)

Fax (34) 916249875

\title{
Trends in distributional characteristics: Existence of global warming*
}

\author{
María Dolores Gadea Rivas ${ }^{\dagger}$ \\ University of Zaragoza
}

\author{
Jesús Gonzalo \\ U. Carlos III de Madrid
}

January, 2017

\begin{abstract}
Does global warming exist? The answer to this question is the starting point for all the other issues involved in climate change analysis. In this paper, global warming is defined as an increasing trend in certain distributional characteristics (moments, quantiles, etc) of global temperatures, and not only on the average. Temperatures are seen as a functional stochastic process from which we obtain distributional characteristics as time series objects. We present a simple robust trend test and prove that it is able to detect the existence of an unknown trend component (deterministic or stochastic) in these characteristics. Applying this trend test to daily Central England temperatures (1772-2016) and to Global cross-sectional temperatures (1880-2015), we obtain the same strong conclusions: (i) there is an increasing trend in all the distributional characteristics (time series and cross-sectional) and this trend is larger in the lower quantiles than in the mean, median and upper quantiles; (ii) there is a negative trend in the characteristics measuring dispersion (lower temperatures approach the median faster than the higher ones).The paper concludes by clearly answering the opening question in the afirmative and showing that global-local warming is not only a phenomenon of an increase in the average temperature but also of a larger increase in the lower temperatures producing a decreasing dispersion. This type of warming has more serious consequences than the one found by using only the average.
\end{abstract}

JEL classification: C31, C32, Q54

Keywords: Climate change; Global-Local warming; Functional stochastic processes; Distributional characteristics; Trends; Quantiles; Temperature distributions

\footnotetext{
* We thank seminar participants at Econometric Models of Climate Change (Aarhus), 11th World Congress of the Econometric Society (Montreal), IAAE (London), Time Series Econometrics Workshop (Zaragoza), CFE-ERCIM (Oviedo), EUI (Firenze), ESSEC (Paris), Loughborough, Notthingham, Nuffield College and INET (Oxford), Erasmus School of Economics (Rotterdam), Manchester, Lancaster and Durham for suggestions. Financial support from the Spanish MINECO (grants ECO2013-46395, ECO2016-78652, ECO2014-58991-C3-1-R and ECO2014-58991-C3-2-R and Maria de Maeztu grant MDM 2014-0431), Bank of Spain (ER grant program), and MadEco-CM (grant S2015/HUM-3444) is gratefully acknowledged.The second author thanks the hospitality of Nuffield College (University of Oxford).

${ }^{\dagger}$ Department of Applied Economics, University of Zaragoza. Gran Vía, 4, 50005 Zaragoza (Spain). Tel:+34 9767 61842, fax: +34 976761840 and e-mail: Igadea@unizar.es

‡ Department of Economics, University Carlos III, Madrid 12628903 Getafe (Spain). Tel: +34 91 6249853, fax: +34 916249329 and e-mail: jesus.gonzalo@uc3m.es
} 


\section{Introduction}

According to the Intergovernmental Panel on Climate Change (IPCC), the study of climate change (CC), and particularly global warming (GW), involves a careful analysis of the following four issues or questions: (i) Existence of GW (what type of GW?); (ii) Causes of GW (is GW caused by human activities?); (iii) Economic effects of GW, and (iv) Economic policies to mitigate these effects. Obviously, without a clear answer to the first question, there is no point in asking the others. The purpose of this paper is to offer a complete answer to the first question: Does GW exist and if so of what type is it? Even if the answer is affirmative, it is important to characterize the type of GW we have because this may influence the analysis of the other three key questions. The existence will be judged by introducing a novel methodology valid for the quantitative analysis of many other important economic issues that require a proper study of trend behaviors: trends in gdp, debt, inequality, etc.

We start by defining GW as an increasing trend in global temperatures. In this paper, trend is understood in a much broader sense than in the literature so far (see White and Granger, 2011). We look for trends in many of the characteristics (moments, quantiles, etc) of the temperature distribution and not only in the average. For instance, a random walk has a trend in the variance but not in the mean. The average temperature might not show any growth pattern but the lower tail might show a clear increase. According to the standard definition in the literature, this would not be interpreted as GW but with our proposal it clearly would. Even when the average shows some growth, to have a wide angle picture of the trending behavior of the whole distribution will help in the analysis of the other three questions in the chain.

There is an extensive literature analyzing the trend behavior (deterministic as well as stochastic) of the mean of temperature distribution [see Harvey and Mills (2003), Hendry and Pretis (2013), Gay-García et al. (2009), Mills (2010), Kauffmann et al. (2006, 2010, 2013), Estrada et al. (2013) and Chang et al. (2015), among others]. It can be said that this approach corresponds to the popular definition of climate: Climate is the average of weather. While our proposal of analyzing the trend behavior of different distributional characteristics agrees more with the climatologists definition: Climate is the statistics of weather. This definition includes not just the average but the statistics of its variability, tail behavior, etc.

For the purpose of this research, global temperatures are seen as a functional 
stochastic process, $X=\left(X_{t}(\omega), t \in T\right)$, where $T$ is an interval in $\mathbb{R}$, defined on a probability space $(\Omega, \Im, P)$ and such that $t \rightarrow X_{t}(\omega)$ belongs to some function space $\mathbf{G}$ for all $\omega \in \Omega$. $X$ defines a $\mathbf{G}$-valued stochastic process. $\mathbf{G}$ can be a Hilbert space as in Bosq (2000) (AR-H model for sequences of random Hilbert functions $\left.X_{1}(\omega), X_{2}(\omega), \ldots, X_{T}(\omega)\right)$, as in Park and Qian (2012) and Chang et al. $(2015,2016)$ (regression models for sequences of random state densities $f_{1}(\omega), f_{2}(\omega), \ldots, f_{T}(\omega)$ ) or a Banach space for sequences of random state distributions $\left(F_{1}(\omega), F_{2}(\omega), \ldots, F_{T}(\omega)\right)$, etc. Instead of modeling the whole sequence of $\mathbf{G}$ functions, as previous authors do, we present an alternative approach where we model certain characteristics, $C_{t}$, of these functions: the state mean, the state variance, the state quantiles, etc. The main advantage of this approach, apart from its simplicity, is that these characteristics become time series objects and, therefore, we can apply all the existing tools in the time series literature for modeling, inference, forecasting, etc. This alternative proposal resembles the quantile curve estimation approach in Draghicescu et al. (2009) and the realized volatility modeling used with high frequency data in financial econometrics (see Andersen et al., 2003, 2006).

In this paper is assumed that, at each period $t$, we have $N$ observations (time series or cross-sectional). From these observations we obtain the characteristics and convert them into time series objects. In order to detect the existence of a trend behavior in any of these characteristics we propose to test $\beta=0$ in the following simple OLS regression, $C_{t}=\alpha+\beta t+u_{t}$. We prove that the $t-t e s t$ is able to detect the standard deterministic trends used in the literature (see Davis, 1941) as well as the stochastic trends generated by long-memory, near unit root or local level models (see Müeller and Watson, 2008).

In order to show the generality of our results, we carry out two types of applications, one with $N$ time series observations for each year $t$ and another with $N$ cross-sectional observations also for each year $t$. The first application studies the trend behavior of the distributional characteristics of the temperature in Central England from January 1st of 1772 to May 31st of 2016. To ensure the robustness of our findings, we also present results for temperatures in other locations: Stockholm, Cadiz and Milan. In the second application, we analyze the global temperature across different stations in the Northern and Southern Hemisphere from 1880 to 2015. Both types of applications lead to similar trend results that can be summarized as follows: (i) there exists a trend in most of the characteristics considered. The trend in the lower quantiles is stronger than in the mean and upper quantiles of 
the temperature distribution. (ii) Dispersion measures like iqr, std and rank show a negative trend. Therefore, we conclude that GW is not only a phenomenon of an increase in the average temperature but also of a larger increase in the lower temperatures producing a decreasing dispersion. Ignoring these facts could have serious consequences in the climate analysis (e.g., acceleration in the global ice melting) and, therefore, they should be taken into account in all future international climate agreements. The actual ones only focus on the mean characteristic.

The rest of the paper is organized as follows. In Section 2, we introduce our definition of GW and of trend. In Section 3, we present our basic framework for time series analysis. In Section 4 we introduce and analyze our proposed trend test $(T T)$ to detect a general unknown trend behavior in any distributional characteristic. Section 5 provides two empirical applications, one with a pure temporal dimension (local daily temperature on an annual basis), and the other with a cross-sectional dimension (global temperatures measured annually station by station). Finally, Section 6 concludes. An Appendix contains detailed proofs of the main results, as well as the finite-sample performance of our proposed test and additional empirical results.

\section{Global Warming and Trends}

In this section, we introduce our definition of global warming as well as the definition of trend that will be used to investigate the existence of GW and to characterize its type.

Definition 1. (Global warming): Global warming is defined as the existence of an increasing trend in some of the characteristics of the global temperature distribution.

As it is mentioned in the Introduction, this definition agrees with the climatologist definition of climate: the statistics of weather. This includes not just the average but the statistics of its variability, tail behavior, etc. The key issue is to find a useful definition and characterization of trend. Surprisingly, there are not many statistic-econometric books that dedicate a chapter to this topic. Maybe the reason is, as Phillips (2005) notes, "No one understands trends, but everyone sees them in the data". Some exceptions are Davis (1941), Anderson (1971) and Kendall and Stuart (1983). Nevertheless, not even in these books a definition or characterization of trend, useful for our GW analysis can be found. We find this in White and Granger (2011) (WG). According to these authors: (i) a trend should have a 
direction; (ii) a trend should be basically smooth; (iii) a trend does not have to be monotonic throughout and (iv) a trend can be a local behavior (observed trends can be related to a particular section of data). These characterizations are formalized by WG in the following two definitions, one for deterministic trends and the other for stochastic trends.

Definition 2. (Deterministic trend (WG, 2011)): Let $\left\{C_{t}\right\}=\left\{C_{t}: t=0,1, \ldots\right\}$ be a sequence of real numbers. If $C_{t} \leq C_{t+1}$ for all $t$, then $\left\{C_{t}\right\}$ is an increasing (weak) trend. If $\left\{-C_{t}\right\}$ is an increasing (weak) trend, then $\left\{C_{t}\right\}$ is a decreasing (weak) trend.

Example of deterministic trend: A polynomial trend for certain values of the $\beta$ parameters $C_{t}=\beta_{0}+\beta_{1} t+\beta_{2} t^{2}+\ldots+\beta_{k} t^{k}$.

More examples can be found in Chapters 1 and 6 of Davis (1941).

Definition 3. Stochastic trend $(W G, 2011))$ : Let $X_{t}$ be a stochastic process.

- Consider $C_{t}=E\left(X_{t}\right)$. If $C_{t}$ is a (weak) increasing trend, then $\left\{X_{t}\right\}$ has a (weak) increasing trend in mean.

- Let $C_{t}=E\left(\left|X_{t}-E\left(X_{t}\right)\right|^{k}\right)$ for finite positive real $k$. If $C_{t}$ is a (weak) increasing trend, then $\left\{X_{t}\right\}$ has a (weak) increasing trend in $k^{\text {th }}$ absolute central moment.

- Let $C_{t}(p)=\inf \left\{x \in \mathbb{R}: F_{t}(x) \geq p\right\}$ be the quantile $p \in(0,1)$ of the distribution function $F_{t}(x)=P\left(X_{t} \leq x\right)$. If $C_{t}(p)$ is a (weak) increasing trend, then $\left\{X_{t}\right\}$ has a (weak) increasing trend in quantile $p$.

Examples of stochastic trends:

- A random walk $X_{t}=X_{t-1}+u_{t}$ has a trend in variance but not in mean

- A random walk with drift $X_{t}=\alpha+X_{t-1}+u_{t}$ has a trend in mean and in variance.

More examples are considered in Müeller and Watson (2008).

Notice that from Definition 3, the concept of stochastic trend considered in the econometrics literature, now becomes a pure deterministic trend in the second moment of the distribution. This implies that by developing a method able to detect deterministic trends and applying this method to different distributional characteristics, we will be able to detect any type of trends. This method will be introduced in section 4. First, we present in the next section the basic framework for our proposed time series analysis to obtain distributional characteristics as time series objects. 


\section{Basic Framework for Time Series Analysis}

In this paper, temperature is contemplated as a functional stochastic process, $X=$ $\left(X_{t}(\omega), t \in T\right)$, where $T$ is an interval in $\mathbb{R}$, defined on a probability space $(\Omega, \Im, P)$ and such that $t \rightarrow X_{t}(\omega)$ belongs to some function space $\mathbf{G}$ for all $\omega \in \Omega$. $X$ defines a $\mathbf{G}$-valued stochastic process.

This function space $\mathbf{G}$ is equipped with a scalar product $<., .>$, and or a norm $\|$.$\| and a Borel \sigma$-algebra, $B_{G}$. It is separable and complete. G can be a Hilbert space as in Bosq (2000) (AR-H model for sequences of random Hilbert functions $\left.X_{1}(\omega), X_{2}(\omega), \ldots, X_{T}(\omega)\right)$, as in Park and Qian (2012), Chang, et al. (2015, 2016) (regression models for sequences of random state densities $f_{1}(\omega), f_{2}(\omega), \ldots, f_{T}(\omega)$ ), a Banach space for sequence of random state distributions $\left(F_{1}(\omega), F_{2}(\omega), \ldots, F_{T}(\omega)\right)$, etc.

A convenient example of an infinite-dimensional discrete-time process consists on associating with $\xi=\left(\xi_{n}, n \in \mathbb{R}_{+}\right)$a sequence of random variables with values in an appropriated function space. This may be obtained by setting

$$
X_{t}(n)=\xi_{t N+n}, 0 \leq n \leq N, t=0,1,2, \ldots, T
$$

so $X=\left(X_{t}, t=0,1,2, \ldots, T\right)$. If the sample paths of $\xi$ are continuous, then we have a sequence $X_{0}, X_{1}, \ldots$ of random variables in the space $C[0, N]$. The choice of the period or segment $t$ is compelling in many concrete situations. In our case, $t$ will be the period of a year, and $N$ could represent temporal or cross-sectional observations.

We can be interested on modeling the whole sequence of $\mathbf{G}$ functions, for instance the sequence of state densities $\left(f_{1}(\omega), f_{2}(\omega), \ldots, f_{T}(\omega)\right)$ as in Chang et al. (2015, 2016) or only certain characteristics $\left(C_{t}(w)\right)$ of these $\mathbf{G}$ functions. For instance, the state mean, the state variance, the state quantile, etc. These characteristics can be considered time series objects and, therefore, all the econometrics tools already developed in the time series literature can be applied to $C_{t}(w)$. It is for this reason that in this paper we follow the second alternative. This alternative resembles the quantile curve estimation analyzed in Draghicescu et al. (2009) and Zhou and Wu (2009). In terms of variance characteristic it also resembles the literature on realized volatility (Andersen et al., 2003, 2006). With this characteristic approach we go from $\Omega$ to $\mathbb{R}^{T}$, as in a standard stochastic process, passing through a $\mathbf{G}$ functional space:

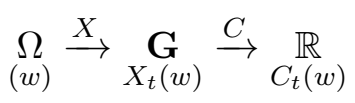


Going back to the convenient example and abusing notation the stochastic structure can be summarized in the following array:

\begin{tabular}{|c|c|c|c|c|}
\hline$X_{10}(w)=\xi_{0}(w)$ & $X_{11}(w)=\xi_{1}(w)$ & $\ldots$ & $X_{1 N}(w)=\xi_{N}(w)$ & $C_{1}(w)$ \\
\hline$X_{20}(w)=\xi_{N+1}(w)$ & $X_{21}(w)=\xi_{N+2}(w)$ & $\ldots$ & $X_{2 N}(w)=\xi_{2 N}(w)$ & $C_{2}(w)$ \\
\hline$\cdot$ & $\cdot$ & $\ldots$ &. &. \\
$\cdot$ &. & $\ldots$ &. &. \\
. &. & $\ldots$ &. &. \\
\hline$X_{T 0}(w)=\xi_{(T-1) N+1}(w)$ & $X_{T 1}(w)=\xi_{(T-1) N+2}(w)$ & $\ldots$ & $X_{T N}(w)=\xi_{T N}(w)$ & $C_{T}(w)$ \\
\hline
\end{tabular}

Through the whole paper, similar to the assumptions in Park and Qian (2012) or Chang et al. (2016), we will assume that in each period $t$ we have enough temporal or cross-section observations $(N \rightarrow \infty)$, such that, these characteristics are consistently estimated.

Assumption 3.1. In each period $t$, the stochastic functional process $X=\left(X_{t}(\omega), t \in\right.$ $T$ ) satisfies certain regularity conditions such that the state densities, distribution and, therefore, quantiles are consistently estimated.

In the temporal framework, local stationarity (Dahlhaus, 2009) plus some strong mixing conditions (Hansen, 2008) are sufficient to obtain uniform strong consistency of suitable regular kernel estimators for the state densities. Local stationarity plus some $\varphi$-mixing conditions (see Degenhardt, et al. 1996) are enough for a central limit theorem to hold for smoothed empirical distribution functions and smoothed sample quantiles for each period or segment $t$. For the cross-sectional situation, similar results hold (see for instance Silverman, 1978) if the state distributions are defined as cross-sectional distributions, and independent and identically distributed observations are available to estimate them for each period.

\section{Testing for a trend}

The objective of this section is to provide a simple test to detect the existence of a general unknown trend component in a given characteristic $C_{t}$ of $X_{t}$. In order to do that we need to convert Definition 3 into a more practical definition:

Definition 4. (Practical definition 1): Let $h(t)$ be a monotonic function of $t$. A characteristic $C_{t}$ of a functional stochastic process $X_{t}$ contains a trend if $\beta \neq 0$ in the regression

$$
C_{t}=\alpha+\beta h(t)+u_{t}
$$


From this definition two questions arise that need to be solved. First, we need to specify which function $h(t)$ to use in regression (3) and second, we have to design a proper test for the null hypothesis of interest, $\beta=0$. Before solving these two questions, the practical Definition 4 requires to introduce some preliminary concepts and results. Specially, the concept of Summability is needed (see Berenguer-Rico and Gonzalo, 2014, for a stochastic version).

Definition 5. (Order of Summability): A trend $h(t)$ is said to be summable of order " $\delta$ " $(S(\delta))$ if there exists a slowly varying function $L(T)^{1}$ s.t.

$$
S_{T}=\frac{1}{T^{1+\delta}} L(T) \sum_{t=1}^{T} h(t)
$$

is $O(1)$ but not $o(1)$.

Some examples to illustrate the order of summability:

Example 4.1. Let $h(t)=c$. Then $\frac{1}{T} \sum_{t=1}^{T} c=c$. Therefore, $\delta=0$.

Example 4.2. Let $h(t)=t^{k}$. Then $\frac{1}{T^{1+k}} \sum_{t=1}^{T} t^{k}=O(1)$. Therefore, $\delta=k$.

Example 4.3. Let $h(t)=e^{\lambda t}$. Then $\frac{1}{e^{\lambda T}} \sum_{t=1}^{T} e^{\lambda t}=O(1)$. Therefore, $\delta_{T}=$ $\frac{\lambda T}{\log (T)}-1$.

Example 4.4. Let $h(t)=\frac{K}{1+B e^{-\lambda t}}$. Then $\frac{1}{T} \sum_{t=1}^{T} \frac{K}{1+B e^{-\lambda t}}=O(1)$. Therefore, $\delta=0$.

Example 4.5. Let $h(t)=\log (t)$. Then $\frac{1}{T \log (T)} \sum_{t=1}^{T} \log (t)=O(1)$. Therefore, $\delta=0$.

Example 4.6. Let $h(t)=\frac{1}{t}$. Then $\frac{1}{\log (T)} \sum_{t=1}^{T} \frac{1}{t}=O(1)$. Therefore, $\delta=-1$.

\footnotetext{
${ }^{1}$ A positive, Lebesgue measurable function $\mathrm{L}$, on $(0, \infty)$ is slowly varying (in Karamata's sense) at $\infty$ if

$$
\frac{L(\lambda n)}{L(n)} \rightarrow 1(n \rightarrow \infty) \forall \lambda>0
$$

(See Embrechts et al., 1999, p. 564). 
The properties of the OLS estimator $\widehat{\beta}$ in regression (3) depend on the balancedness between the trend components of the dependent variable $C_{t}$ and the regressor $h(t)$. To characterize this balancedness we need the following definition:

Definition 6. (Trend strength): A trend function $h(t)$ is said to be stronger than another trend function $g(t)$ if $\delta_{h}>\delta_{g}$.

Now, with Definitions 5 and 7 we have all the necessary elements to present our trend test to detect a general trend component in a given characteristic $C_{t}=$ $h(t)+I(0),{ }^{2}$ with $h(t)$ unknown. But first, let us remind a very well known related result (see chapter 16 in Hamilton, 1994):

Proposition 1. Let $C_{t}=I(0)$. In the regression

$$
C_{t}=\alpha+\beta t+u_{t}
$$

the OLS estimator

$$
\widehat{\beta}=\frac{\sum_{t=1}^{T}\left(C_{t}-\bar{C}\right)(t-\bar{t})}{\sum_{t=1}^{T}(t-\bar{t})^{2}}
$$

satisfies

$$
T^{3 / 2} \widehat{\beta}=O_{p}(1)
$$

and asymptotically $(T \rightarrow \infty)$

$$
t_{\beta=0} \text { is } N(0,1) .
$$

Proposition 2. Let $C_{t}=h(t)+I(0)$ such that $h(t)$ is $S(\delta)$ with $\delta \geq 0$, and such that the function $g(t)=h(t) t$ is $S(\delta+1)$. In the regression

$$
C_{t}=\alpha+\beta t+u_{t}
$$

the OLS $\widehat{\beta}$ estimator satisfies

$$
T^{(1-\delta)} \widehat{\beta}=O_{p}(1)
$$

In order to analyze the behavior of the $t$-statistic $t_{\beta}=0$, we will assume that the function $h(t)^{2}$ is $S(1+2 \delta-\gamma)$ with $0 \leq \gamma \leq 1+\delta$. Then,

$$
t_{\beta=0}=\left\{\begin{array}{l}
O_{p}\left(T^{\gamma / 2}\right) \text { for } 0 \leq \gamma \leq 1 \\
O_{p}\left(T^{1 / 2}\right) \text { for } 1 \leq \gamma \leq 1+\delta
\end{array}\right.
$$

\footnotetext{
${ }^{2}$ Our definition of an I(0) process follows Johansen (1995). A stochastic process $Y_{t}$ which satisfies $Y_{t}-E\left(Y_{t}\right)=\sum_{i=1}^{\infty} \Psi_{i} \varepsilon_{t-i}$ is called $\mathrm{I}(0)$ if $\sum_{i=1}^{\infty} \Psi_{i} z^{i}$ converges for $|z|<1$ and $\sum_{i=1}^{\infty} \Psi_{i} \neq 0$, the condition $\varepsilon_{t} \sim \operatorname{iid}\left(0, \sigma^{2}\right)$ being understood.
} 
Proof in the Appendix.

The following few examples illustrate how to use Proposition 2.

Example 4.7. Let $C_{t}=h(t)+I(0)$, with $h(t)=t^{2}$. The summability parameters are $\delta=2$ and $\gamma=1$. Then, in regression (9), $\widehat{\beta}$ and $t_{\beta=0}$ diverges as $T \rightarrow \infty$.

Example 4.8. Let $C_{t}=h(t)+I(0)$, with $h(t)=t$. The summability parameters are $\delta=1$ and $\gamma=1$. Then, in regression (9), $\widehat{\beta}=O_{p}(1)$; but $t_{\beta=0}$ diverges as $T \rightarrow \infty$.

Example 4.9. Let $C_{t}=h(t)+I(0)$, with $h(t)=t^{1 / 2}$. The summability parameters are $\delta=1 / 2$ and $\gamma=1$. Then, in regression (9), $\widehat{\beta} \stackrel{p}{\rightarrow} 0$; but $t_{\beta=0}$ diverges as $T \rightarrow \infty$.

Example 4.10. Let $C_{t}=h(t)+I(0)$, with $h(t)=\log (t)$. The summability parameters are $\delta=0$ and $\gamma=1$. Then, in regression $(9), \widehat{\beta} \stackrel{p}{\rightarrow} 0$; but $t_{\beta=0}$ diverges as $T \rightarrow \infty$.

Example 4.11. Let $C_{t}=h(t)+I(0)$, with $h(t)=e^{\lambda t}$. The summability parameters are are $\delta_{T}=\frac{\lambda T}{\log (T)}-1$ and $\gamma=0$.. Then, in regression (9), $\widehat{\beta}$ diverges and $t_{\beta}=0=O_{p}(1)$ as $T \rightarrow \infty$. It can be proved that asymptotically $t_{\beta}=0 \geqslant \mathbf{z}_{0.95}$, for $\lambda \in(0,2.095)$.

Example 4.12. Let $C_{t}=h(t)+I(0)$, with $h(t)=\frac{K}{1+B e^{-\lambda t}}$. The summability parameters are $\delta=0$ and $\gamma=1$. Then, in regression $(9), \widehat{\beta} \stackrel{p}{\rightarrow} 0$; but $t_{\beta=0}$ diverges as $T \rightarrow \infty$.

A question of large empirical relevance is how does our trend test of Proposition 2 behaves when $C_{t}=I(1)$. Following Durlauf and Phillips (1988), $T^{1 / 2} \widehat{\beta}=O_{p}(1)$; but $t_{\beta=0}$ diverges as $T \rightarrow \infty$. Therefore, our trend test will be able to detect the stochastic trend generated by an I(1) process. In fact, our test will detect the trends generated by any of the three standard persistent processes considered in the literature (see Muller and Watson, 2008): (i) fractional or long-memory model; (ii) near unit root AR model and (iii) local level model. Let

$$
C_{t}=\mu+z_{t}, t=1, \ldots, T
$$


In the first model, $z_{t}$ is a fractional process with $1 / 2<d<3 / 2$. In the second model, $z_{t}$ follows an AR with its largest root close to unity, $\rho_{T}=1-c / T$. For the third one, $z_{t}$ is decomposed into an $\mathrm{I}(1)$ plus and $\mathrm{I}(0)$ component. Its simplest format would be, $z_{t}=v_{t}+\epsilon_{t}$ with $v_{t}=v_{t-1}+\eta_{t}$ where $\epsilon_{t}$ is $I D\left(0, q * \sigma^{2}\right), \eta_{t} I D\left(0, \sigma^{2}\right)$ and both disturbances are serially and mutually independent. Notice that the pure unit root process $(I(1))$ is nested in all the three models: $d=1$, c=0, or $q=0$.

The long-run properties implied by each of these models can be characterized by the stochastic properties of the partial sum process for $z_{t}$. Standard assumptions considered in the macroeconomics or finance literature assume the existence of a " $\delta$ " such that $T^{-1 / 2+\delta} \sum_{t=1}^{T} z_{t} \longrightarrow \sigma H($.$) , where " \delta$ " is a model-specific constant and $H$ is a model-specific mean-zero Gaussian process with a given covariance kernel $k(r, s)$. Then, is clear that the process $C_{t}=\mu+z_{t}$ is summable of order " $\delta$ " (see Berenguer-Rico and Gonzalo, 2014). This is the main reason, Proposition 3 holds for these three persistent processes. Next proposition formalizes this result.

Proposition 3. Let $C_{t}=\mu+z_{t}, t=1, \ldots, T$, with $z_{t}$ any of the following three processes: (i) fractional or long-memory model with $1 / 2<d<3 / 2$; (ii) near unit root $A R$ model and (iii) local level model, and such that $T^{-1 / 2+\delta} \sum_{t=1}^{T} z_{t} \longrightarrow \sigma H($.) where " $\delta$ " is a model-specific constant and $H$ is a model-specific mean-zero Gaussian process with a given covariance kernel $k(r, s)$. Then, in the regression

$$
C_{t}=\alpha+\beta t+u_{t}
$$

the t-statistic

$$
t_{\beta=0}=O_{p}\left(T^{1 / 2}\right)
$$

Proof in the Appendix.

Summarizing, Propositions 2 and 3 imply that Definition 4 can be simplified in the following practical definition:

Definition 7. (Practical definition 2): A characteristic $C_{t}$ of a functional stochastic process $X_{t}$ contains a trend if $\beta \neq 0$ in the regression

$$
C_{t}=\alpha+\beta t+u_{t} .
$$

It is important to highlight two facts related to the possible serial correlation present in $u_{t}$ : (i) From the classical result of Grenander and Rosenblatt (1957) it is well-known that the OLS $\widehat{\beta}$ estimator in regression (13) is asymptotically equivalent to the GLS estimator. (ii) In practice, it is recommended to use a HAC version of $t_{\beta=0}$ (see Canjels and Watson, 1997 and Vogelsang, 1998). 
Appendix includes a detailed analysis of the finite-sample performance of this test for several types of deterministic and stochastic trends.

\section{Local and global warming: time series and cross- sectional data}

It is convenient to start this section by reminding the type of data structure we analyze in this paper, in order to answer the first question of any climate change study: Does global warming exist? and if so, of which type is it? Following the convenient example (see (2) in section 3), $X$ will be local or global temperature; $T$ (number of periods) will be years; $N$ will have a temporal structure (days) or a cross-sectional dimension (stations in both Hemispheres) and $C_{t}=\left(C_{1 t}, C_{2 t}, \ldots, C_{p t}\right)$ will be a vector of $p$ distributional characteristics (mean (mean), maximum (max), minimum ( $\mathrm{min})$, standard deviation $(s t d)$, interquartile range $(i q r)$, total range (rank), kurtosis (kur), skewness (skw) and the following quantiles: q5, q10, q20, $q 30, q 40, q 50, q 60, \mathrm{q} 70, q 80, q 90$ and $q 95)$ estimated from $N$ observations.

In this section we implement our trend test (Proposition 2 and 3 ) to two types of data: (i) Time series data (Central England temperatures, $N$ days and $T$ from 1772 to 2016) and (ii) Cross-sectional data (Global Earth temperature, $N$ stations and $T$ from 1880 to 2015).

In the rest of the section, we present a brief description of the data, a unit root analysis and apply our trend test to detect the existence of local and/or global warming.

\subsection{Time series data: local warming}

The longest temperature record series (thermometer measured) runs from 1659 to the present. This data is monthly and annual from England. There is also daily temperature data from 1772. However, there is no instrumental data before 1659 because the thermometer was only invented a few decades earlier. These data were originally published by Gordon Manley in 1953 in a database called The Central England Temperature (CET) and provided monthly mean surface air temperatures, for the Midlands region of England, in degrees Celsius from the year 1659 to the present (Manley, 1953, 1974). Parker et al. (1992) builds a daily version of the database from 1772 to the present day, which is updated continuously. They evaluate 
recent urban warming influences and correct the series after $1974 .{ }^{3}$ Figure 1 shows the yearly, monthly and daily version of the data. ${ }^{4}$

The advantage of this climate database from CET is double, its length and its high frequency. In particular, having daily observations for each year (1772-2016) allow us to compute the different distributional characteristics of interest and convert them into time series objects. Figure 2 presents the annual densities from 1772 to 2016 and Figure 3 shows the path of these characteristics.

More recently, a European Union research project (IMPROVE) studied the past climatic variability using early daily European instrumental sources. This project collected records of temperature in different European areas, from the Baltic to the Mediterranean and from the Atlantic to Eastern Europe. IMPROVE's general objectives were to assess correction and homogenisation protocols for early daily instrumental records of air temperature and air pressure but the quality and continuity of the series are very heterogeneous and only the Swedish series (Stockolm) has continued to be updated by Anders Moberg. ${ }^{5}$ In addition to Stockolm we have selected data form Cadiz and Milan to have assurance of the robustness of our CET results. The density of the data (Figure 7) and the econometric results for these additional data are shown in the Appendix.

\subsubsection{Results}

Before testing the presence of trends in the distributional characteristics of CET data, we test for the existence of unit roots. For that, we use the well-known Augmented Dickey-Fuller test (ADF, Dickey and Fuller, 1979) where the number of lags is selected in accordance with SBIC criterion. ${ }^{6}$ The results displayed in Table 1 show that we can reject the null hypothesis of a unit root for all the characteristics considered.

The presence of a trend in temperature characteristics is tested by applying the proposed $T T$ in regression (13). This regression has been estimated by OLS-

\footnotetext{
${ }^{3}$ Some relevant references for better understanding of these series are Parker et al. (1992), Manley (1953, 1974) and Parker and Horton (2005). The data are available in http://www.metoffice.gov.uk/hadobs/hadcet/.

${ }^{4}$ Some analysis of the statistical properties of annual and monthly averages of these data can be found in Harvey and Mills (2003) and Proietti and Hillebrand (2016).

${ }^{5}$ They can be advised with the Bolin Center for Climate Research: http://bolin.su.se/data/stockholm/.

${ }^{6}$ The number of selected lags is low in general. Other information criterion, such as Akaike or methods, like the general-to-specific one, yield a higher number of lags, but the conclusions about the stationarity of the series is the same.
} 
HAC and the results are displayed in Table 2, showing a significant trend in all characteristics. The trend is positive in all series with the exception of those that measure volatility ( $s t d, i q r$, rank), which have a significant negative trend. With respect to the rest, the mean has a trend coefficient of 0.0038 , which implies an increase of 0.4 degrees after 100 years, and we find the highest positive trends in the lower quantiles. Notice that the trend coefficient of quantiles ranges from 0.0072 in quantile $5 \%(q 5)$ to 0.0013 in quantile $80 \%$ ( $q 80)$. If should be noted that this test only confirms the existence of a trend, but says nothing about its nature. This would require an additional empirical study, looking for the most suitable type of trend and this is beyond the scope of this paper.

These results are very suggestive and illustrate the usefulness of our proposal of analyzing a wide set of distributional characteristics of the temperature instead of only the mean. To strengthen this idea, we test for co-trending in different sets of characteristics. The results of applying the Wald test to different co-trending possibilities appear in Table 3. We see that the null hypothesis of co-trending in all quantiles is rejected. Nevertheless, this is not the case if we test the null of equal trends in groups of quantiles, namely, lower, medium and upper. Finally, to complete this study, we test the existence of trend in some important spacing characteristics and find that the difference between the lowest quantile, $q 5$, and the median shows a decreasing and significant trend, but the difference between the highest quantile, $q 95$, and the median, does not show any trend. The conclusion is, again, that the minimum temperatures have approached the median more rapidly than the maximum temperatures have distanced themselves from it, contrary to what is usually thought.

To close this section we carry out a parallel study with temperature data for the other cities mentioned previously: Stockholm, Cadiz and Milan (See Figure 7). Results in Table 11 for unit roots and the trend analysis in Tables 12, 13 and 14 lead to the same conclusions. Summing up our findings, we have identified patterns in the distributional characteristics of temperatures that are common for different cities with different geographic positions, inferring that this maybe a global phenomenon. Next section investigates exactly this conjecture.

\subsection{Cross-sectional data: Global warming}

The Climate Research Unit (CRU) offers monthly and yearly data of land and sea temperatures in both hemispheres from 1850 to the present collected in different 
stations distributed around the world ${ }^{7}$. Each station temperature is converted to an anomaly taking 1961-90 as the base period, ${ }^{8}$ and each grid-box value, on a 5 degree grid, is the mean of all the station anomalies within that grid box. ${ }^{9}$ This database, and specially the annual temperature of the Noth-Hemispheric, has become one of the most popular to illustrate the global warming from the graph known as the "hockey stick", frequently used by academics and other institutions, such as, the IPCC. In this paper, we prefer to base our analysis in station raw data (see density in Figure 4). These data show high variability at the beginning of the time-period, probably due to the scarce number of stations in this early stage of the project, as Jones et al. (2012) pointed out. Following these authors we start at 1880.

The construction of characteristics deserves a little of attention. Although the total number of stations recorded is over 7,000, the effective number fluctuates each year. It reaches a minimum in 1850 and a maximum during the 1951-2010 period. Furthermore, its geographic distribution is not homogeneous. Coverage is denser over the more populated parts of the world, particularly, the United States, Southern Canada, Europe and Japan, and sparsest over the interior of the South American and African continents and over Antarctica. This provokes a disequilibrium of the Northern Hemisphere (NH) with respect to the Southern Hemisphere (SH). To guarantee the stability of the characteristics over whole sample, we have selected only stations with data for all years, which forces us to reduce the sample size. With this procedure, and starting in 1880 , we have $N=249$ stations. Figure 5 shows the

\footnotetext{
${ }^{7}$ HadCRUT4 is a global temperature dataset, providing gridded temperature anomalies across the world as well as averages for the hemispheres and the globe as a whole. CRUTEM4 and HadSST3 are the land and ocean components of this overall dataset, respectively. These datasets have been developed by the Climatic Research Unit (University of East Anglia) in conjunction with the Hadley Centre (UK Met Office), apart from the sea surface temperature (SST) dataset which was developed solely by the Hadley Centre. We have used the CRUTEM 4.5.0.0 version that can be downloaded in (https://crudata.uea.ac.uk/cru/data/temperature/). A recent revision of the methodology can be found in Jones et al. (2012).

${ }^{8}$ To avoid biases that could result from the different elevations of stations, monthly average temperatures are reduced to anomalies from the period with best coverage (1961-90). Because many stations do not have complete records for the 1961-90, the are estimated from neighbouring records or using other sources of data.

${ }^{9}$ Today there are other many institutions that collect information on climate and temperature data. Among them we can mention: The National Oceanic and Atmospheric Administration (NOAA) presents daily and monthly raw data of temperatures classified by countries and stations from 1961 to the present. The National Aeronautics and Space Administration (NASA) offers monthly raw data for stations, anomalies for countries and a method of homogenization of station data since 1880. Finally, Berkeley University offers monthly raw data for stations and anomalies for countries for land temperatures since 1750, for land and ocean temperatures since 1850 and experimental daily land data since 1880.
} 
distributional characteristics as time series objects.

This method of building characteristics has a consequence that it should be mentioned: the mean calculated with filtered raw data does not exactly match with the reported by the CRU, which is calculated, for each hemisphere, as the weighted average of all the non-missing, grid-box anomalies in each hemisphere. The weights used are the cosines of the central latitudes of each grid box and the global average for is a weighted average of the $\mathrm{NH}$ and $\mathrm{SH}$. The weights are 2 for the $\mathrm{NH}$ and one for the SH. Figure 8 shows the path of both hemispheres and global temperatures at monthly and annual frequency. This circumstance leads us to carry out an additional complementary study with data grids (see the Appendix).

Summarizing, in the main text of the paper we analyze raw global data (stations instead of grids) from 1880 to 2015, and for homogeneity reasons we only use data from the stations represented in the whole sample period. An equivalent grid data analysis is shown in the Appendix.

\subsubsection{Results}

The ADF test rejects the null hypothesis of a unit root for all the characteristics (see Table 4), with the only exception of $q 80$. The unit root analysis has been completed in two ways. First, we have applied the ADF test station by station and counting the number of rejections and, second, we have carried out a battery of panel unit root tests. Results in Table 5 reinforce the conclusions of no unit roots in the temperature process station by station.

As Tables 15 and 16 show, similar conclusions are obtained when we test the null hypothesis of a unit root with distributional characteristics obtained from the grids (see the Appendix). ${ }^{10}$ The picture changes if we include all the existing grids in each year. In this case, we can not reject the null of a unit root in many characteristics, including the mean. ${ }^{11}$ This is consistent with the widespread belief that the global temperature has a unit root, a result that comes from the analysis of the annual mean temperature in the northern hemisphere. At the bottom of Table 15 we present results for monthly and annual average series provided by CRU. The ADF test rejects the null for the three monthly series, Global, NH and SH, but it does not

\footnotetext{
${ }^{10}$ These computed in the same way than from stations. Therefore, we have only considered grids that are represented in the 1880-2015 sample. This yields a total of 160 grids.

${ }^{11}$ With the same data and following a pure functional approach, Chang et al. (2015) find certain evidence of unit root behavior in some moments. Nevertheless, a panel unit root test with all the grids rejects the unit root hypothesis. Results available upon request.
} 
for the annual Global and NH average temperatures. These last time series are the most commonly used in the literature to show evidence of global warming. The high concentration of stations in the NH, could imply that the final series suffers a spatial and temporal aggregation effect that increases artificially the persistence (see Taylor, 2001). Other global surface temperatures like those used in Kaufmann et al. (2006, 2010, 2013) also show evidence of unit roots, but Gay-García et al. (2009), Estrada et al. (2013) attribute the non-stationarity to the presence of structural breaks. In any case, our $T T$ is able to detect trends even if they have been generated from unit root processes (see Proposition 3).

Finally, our $T T$ is applied to the characteristics calculated from the described cross-sectional data. The results, displayed in Table 6 lead to the same conclusions obtained with characteristics from time series data. ${ }^{12}$ This similarity is evident in Figure 6 which compares the trend coefficient estimated of both analysis, time and cross-sectional. This finding endorses the behavior patterns of temperature distribution as a global phenomenon. To sum up, we find a trend in most of the $C_{i t}$ $(i=1, \ldots, p)$ considered, stronger for lower quantiles than for the mean and upper quantiles. Dispersion measures like $i q r$, std and rank show a negative trend. 13 Therefore, we conclude that global warming is not only a phenomenon of an increase in the average temperature but also of a larger increase in the lower temperatures producing a decreasing dispersion.

\section{Conclusions}

This paper introduces a novel approach to model the time evolution of certain distributional characteristics of a functional stochastic process (moments, quantiles, etc). This is possible because these distributional characteristics can be obtained as time series objects and, therefore, all the existing tools (modeling, inference, forecasting, etc) available in the time series literature can be applied to them. In particular, we present a simple robust trend test that is able to detect unknown trend components in any of these characteristics.

By defining global warming as the existence of an increasing trend in some of the characteristics of the temperature distribution, testing for a trend on them is equivalent to testing for the existence of global warming. We apply our methodology

\footnotetext{
${ }^{12}$ This similitude can be extended to the analysis of co-trending, (Table 7), although we reject that upper quantiles have the same trend coefficients at $5 \%$ significant level.

${ }^{13}$ Similar results are obtained using grid data, see Tables 17 and 18.
} 
to two types of data: (i) Central England, time series distributional characteristics and (ii) Global temperature, cross-sectional distributional characteristics. In both cases, we obtain the same conclusions: (i) there is a trend component in all the distributional characteristics of interest, and this trend is stronger in lower quantiles than in the mean, median and upper quantiles, and (ii) the distributional characteristics capturing the dispersion of the temperature have a negative trend (lower quantiles evolve toward the median faster than the upper quantiles). Therefore, there is clear evidence of local-global warming. This warming is stronger in the lower temperatures than in the rest of the distribution. This result can have very serious consequences, the misunderstanding of phenomena such as ice melting is one of them. Future international climate agreements should take this into consideration and not only focus on the mean temperature.

Notice that our trend test is able to detect the existence of an unknown trend; but not the concrete type of the trend component. Further research will go exactly in this direction: Modeling the right type of trend and developing methods to forecast this trend component.

\section{References}

[1] Andersen, T.G., Bollerslev, T., Diebold, F.X., Labys, P., 2003. Modeling and forecasting realized volatility. Econometrica 71, 529-626.

[2] Andersen, T.G., Bollerslev, T., Christoffersen, P., Diebold, F.X., 2006. Volatility and correlation forecasting. In: Elliott, G., Granger, C.W.J., Timmermann, A., eds. Handbook of Economic Forecasting. Amsterdam: North-Holland, 778878.

[3] Anderson, T.W., 1971. The Statistical Analysis of Time Series. John Wiley \& Sons, Inc.

[4] Berenguer-Rico, V., Gonzalo, J., 2014. Summability of Stochastic Processes- A generalization of integration and co-integration valid for non-linear processes. Journal of Econometrics 178, 331-341.

[5] Bosq, D., 2000. Linear Processes in Function Spaces. Lecture Notes in Statistics 149. New York: Springer. 
[6] Breitung, J., 2000. The Local Power of Some Unit Root Tests for Panel Data, in B. Baltagi (ed.), Advances in Econometrics, Vol. 15: Nonstationary Panels, Panel Cointegration, and Dynamic Panels, Amsterdam: JAI Press, 161-178.

[7] Canjels, E., Watson, M.W., 1997. Estimating Deterministic Trends in the Presence of Serially Correlated Errors. The Review of Economics and Statistics 79, 184-200.

[8] Chang, Y., Kim, Ch.S., Miller, J.I., Park, J.Y., Park, S., 2015. Time Series Analysis of Global Temperature Distributions: Identifying and Estimating Persistent Features in Temperature Anomalies. Working Paper 15-13, University of Missouri.

[9] Chang, Y., Kim, Ch.S, Park, J.Y., 2016. Nonstationarity in Time Series of State Densities. Journal of Econometrics 192, 152-167.

[10] Choi, I., 2001. Unit Root Tests for Panel Data. Journal of International Money and Finance 20, 249-272.

[11] Dahlhaus, R., 2009. Local Inference for Locally Stationary Time Series Based on the Empirical Spectral Measure. Journal of Econometrics 151, 101-112.

[12] Davis, H. 1941. The Analysis of Economic Time Series, The Principia Press.

[13] Degenhardt, H.J.A., Puri, M.L., Sun, S., Van Zuijlen, M.C.A., 1996. Characterization of weak convergence for smoothed empirical and quantile processes under $\varphi$-mixing. Journal of Statistical Planning and Inference 53, 285-295.

[14] Dickey, D.A., Fuller, W.A., 1979. Distribution of the Estimators for Autoregressive Time Series With a Unit Root. Journal of the American Statistical Association 74, 427-431.

[15] Draghicescu, D., Guillas, S., Wu, W.B., 2009. Quantile Curve Estimation and Visualization for Nonstationary Time Series. Journal of Computational and Graphical Statistics 18, 1-20.

[16] Durlauf, S.N., Phillips, P.C.B., 1988. Trends versus random walks in time series analysis. Econometrica 56, 1333-1354.

[17] Embrechts, P., Klüppelberg, C., Mikosh, T., 1999. Modelling Extremal Events for Insurance and Finance. Springer-Verlag, Berlin. 
[18] Estrada, F., Perron, P, Gay-García C., Martínez-López, B. 2013. A Time-Series Analysis of the 20th Century Climate Simulations Produced for the IPCCs Fourth Assessment Report. PLoS ONE 8, e60017.

[19] Gay-García C, Estrada F, Sánchez A., 2009. Global and hemispheric temperatures revisited. Climate Change 94, 333-349.

[20] Grenander, U., Rosenblatt, M. 1957. Statistical Analysis of Stationary Time Series. New York: Wiley.

[21] Hamilton, J., 1994. Time Series Analysis. Princeton University Press.

[22] Hansen, B.E., 2008. Uniform Convergence Rates for Kernel Estimation with Dependent Data. Econometric Theory 24, 726-748.

[23] Harvey, D.I., Mills, T.C., 2003. Modelling Trends in Central England Temperatures. Journal of Forecasting 22, 35-47.

[24] Hendry, D.F., Pretis, F. 2013. Some Fallacies in Econometric Modelling of Climate Change. Department of Economics. Oxford University. Discussion Papers 643.

[25] Im, K.S., Pesaran, M.H., Shin, Y., 2003. Testing for Unit Roots in Heterogeneous Panels. Journal of Econometrics, 115, 53-74.

[26] Johansen, S., 1995. Likelihood-based Inference in Cointegrated Vector Autoregressive Models. Oxford: Oxford University Press.

[27] Jones, P.D., Lister, D.H., Osborn, T.J., Harpham, C., Salmon, M., Morice, C.P., 2012. Hemispheric and large-scale land surface air temperature variations: an extensive revision and an update to 2010. Journal of Geophysical Research $117,1-29$.

[28] Kaufmann, R.K., Kauppi, H., Stock, J., 2006. Emissions, Concentrations \& Temperature: A Time Series Analysis. Climatic Change 77, 249-278.

[29] Kaufmann, R.K., Kauppi, H., Stock, J., 2010, Does temperature contain a stochastic trend? Evaluating conflicting statistical results. Climatic Change 101, 395-405. 
[30] Kaufmann, R.K., Kauppi, H., Mann, M.L., Stock, J., 2013. Does temperature contain a stochastic trend: linking statistical results to physical mechanisms, Climatic Change 118, 729-743..

[31] Kendall, M.G., Stuart, A., 1983. Advanced Theory of Statistics, Hafner Press, New York.

[32] Levin, A., Lin, C.F., Chu, C., 2002. Unit Root Tests in Panel Data: Asymptotic and Finite-Sample Properties. Journal of Econometrics 108, 1-24.

[33] Maddala, G.S., Shaowen, W. 1999. A Comparative Study of Unit Root Tests with Panel Data and a New Simple Test. Oxford Bulletin of Economics and Statistics 61, 631-652.

[34] Maddison, A., 2013. The Maddison-Project, http://www.ggdc.net/maddison/ maddison-project/home.htm, 2013 version.

[35] Manley, G. 1953. The mean temperature of Central England, 1698 to 1952. Quarterly Journal of the Royal Meteorological Society 79, 242-261.

[36] Manley, G. 1974. Central England Temperatures: monthly means 1659 to 1973. Quarterly Journal of the Royal Meteorological Society 100, 389-405.

[37] Marmol, F., Velasco, C., 2002. Trend stationarity versus long-range dependence in time series analysis. Journal of Econometrics 108, 25-42.

[38] Mills, T.C., 2010. Skinning a cat: alternative models of representing temperature trends. An editorial comment. Climate Change 101, 415-426.

[39] Müeller, U.K., Watson, M. 2008. Testing models of low-frecuency variability. Econometrica 76, 979-1016.

[40] Park, J.Y., Qian, J. 2012. Functional Regression of Continuous State Distributions. Journal of Econometrics 167, 397-412.

[41] Parker, D.E., Legg, T.P., Folland, C.K., 1992. A new daily Central England Temperature Series, 1772-1991. International Journal of Climatology 12, 317342 .

[42] Parker, D.E., Horton, E.B., 2005. Uncertainties in the Central England Temperature series since 1878 and some changes to the maximum and minimum series. International Journal of Climatology 25, 1173-1188. 
[43] Phillips, P. 2005. Challenges of trending time series econometrics. Mathematics and Computers in Simulation 68, 401-416.

[44] Proietti, T., Hillebrand, H., 2016. Seasonal changes in central England temperatures. Journal of the Royal Statistics Society. Statistics in Society. Series A. (forthcoming).

[45] Silverman, B.W., 1978. Weak and Strong Uniform Consistency of the Kernel Estimate of a Density and its Derivatives. The Annals of Statistics 6, 177-184.

[46] Taylor, A., 1981. Potential Pitfalls for the Purchasing-Power-Parity Puzzle? Sampling and Specification Biases in Mean-Reversion Tests of the Law of One Price. Econometrica 69, 473-498.

[47] Vogelsang, T.J., 1998. Trend Function Hypothesis Testing in the Presence of Serial Correlation. Econometrica 66, 123-148.

[48] White, H., Granger, C.W.J., 2011. Consideration of Trends in Time Series. Journal of Time Series Econometrics 3, 1-4.

[49] Zhou, Z., Wu, W.B., 2009. Local linear quantiles estimation for nonstarionary time series. The Annals of Statistics 37, 2696-2729. 


\section{Tables}

Table 1

ADF unit root test (CET data)

\begin{tabular}{lccc}
\hline \hline Characteristic & ADF-SBIC & p-value & lags \\
\hline mean & -8.58 & 0.000 & 1 \\
max & -14.86 & 0.000 & 0 \\
min & -15.15 & 0.000 & 0 \\
std & -15.93 & 0.000 & 0 \\
iqr & -16.06 & 0.000 & 0 \\
rank & -16.30 & 0.000 & 0 \\
kur & -16.35 & 0.000 & 0 \\
skw & -13.19 & 0.000 & 0 \\
q5 & -14.30 & 0.000 & 0 \\
q10 & -14.36 & 0.000 & 0 \\
q20 & -14.46 & 0.000 & 0 \\
q30 & -14.77 & 0.000 & 0 \\
q40 & -14.38 & 0.000 & 0 \\
q50 & -14.10 & 0.000 & 0 \\
q60 & -12.89 & 0.000 & 0 \\
q70 & -13.66 & 0.000 & 0 \\
q80 & -14.56 & 0.000 & 0 \\
q90 & -14.38 & 0.000 & 0 \\
q95 & -14.91 & 0.000 & 0 \\
\hline \hline
\end{tabular}

Notes: Annual distributional characteristics of temperature from daily Central England data (1772-2016). Lag-selection according to SBIC criterion. 
Table 2

Trend test (CET data)

\begin{tabular}{lcc}
\hline \hline Characteristic & Coeff & p-value \\
\hline mean & 0.0038 & 0.0000 \\
max & 0.0033 & 0.0053 \\
min & 0.0110 & 0.0000 \\
std & -0.0020 & 0.0000 \\
iqr & -0.0043 & 0.0000 \\
rank & -0.0077 & 0.0000 \\
kur & 0.0004 & 0.0514 \\
skw & 0.0003 & 0.0438 \\
q5 & 0.0072 & 0.0000 \\
q10 & 0.0067 & 0.0000 \\
q20 & 0.0060 & 0.0000 \\
q30 & 0.0052 & 0.0000 \\
q40 & 0.0044 & 0.0000 \\
q50 & 0.0034 & 0.0000 \\
q60 & 0.0023 & 0.0050 \\
q70 & 0.0015 & 0.0482 \\
q80 & 0.0013 & 0.0621 \\
q90 & 0.0016 & 0.0600 \\
q95 & 0.0021 & 0.0262 \\
\hline \hline
\end{tabular}

Notes: Annual distributional characteristics of temperature from daily Central England data (1772-2016). OLS estimates and HAC $t_{\beta=0}$ from regression: $C_{t}=$ $\alpha+\beta t+u_{t}$. 
Table 3

Co-trending analysis (CET data)

\begin{tabular}{|c|c|c|}
\hline Characteristic & Wald test & $\mathrm{p}$-value \\
\hline \multicolumn{3}{|l|}{ QUANTILES } \\
\hline All quantiles (q5, q10,..,q90,q95) & 38.505 & 0.000 \\
\hline Lower quantiles (q5-q30) & 1.474 & 0.688 \\
\hline Medium quantiles (q40-q60) & 2.450 & 0.294 \\
\hline Upper quantiles (q70-q95) & 0.283 & 0.963 \\
\hline \multicolumn{3}{|l|}{ SPACING } \\
\hline & Trend-coeff. & p-value \\
\hline q50-q5 & -0.003 & 0.004 \\
\hline q95-q50 & -0.001 & 0.159 \\
\hline
\end{tabular}

Notes: Annual distributional characteristics (quantiles) of temperature from daily Central England data (1772-2016). At the top panel, Wald test of the null hypothesis of equality of trend coefficients of a given set of characteristics. P-values calculated from bootstrap critical values. At the bottom, the $T T$ is applied to the difference between two representative quantiles. 
Table 4

ADF unit root test (CRU station data)

\begin{tabular}{lccc}
\hline \hline Characteristic & ADF-SBIC & p-value & lags \\
\hline mean & -8.35 & 0.000 & 0 \\
max & -6.06 & 0.000 & 3 \\
min & -9.04 & 0.000 & 0 \\
std & -8.88 & 0.000 & 0 \\
iqr & -10.41 & 0.000 & 0 \\
rank & -9.32 & 0.000 & 0 \\
kur & -4.29 & 0.005 & 3 \\
skw & -10.94 & 0.000 & 0 \\
q5 & -10.22 & 0.000 & 0 \\
q10 & -5.13 & 0.000 & 4 \\
q20 & -8.89 & 0.000 & 0 \\
q30 & -9.41 & 0.000 & 0 \\
q40 & -9.40 & 0.000 & 0 \\
q50 & -9.49 & 0.000 & 0 \\
q60 & -9.23 & 0.000 & 0 \\
q70 & -9.40 & 0.000 & 0 \\
q80 & -2.36 & 0.414 & 3 \\
q90 & -6.82 & 0.000 & 0 \\
q95 & -5.07 & 0.000 & 0 \\
\hline \hline
\end{tabular}

Notes: Annual distributional characteristics calculated from CRU station data (1880-2015). Lag-selection according to SBIC criterion. 
Table 5

Additional unit root analysis (CRU station data)

\begin{tabular}{lrc}
\hline \hline \multicolumn{3}{c}{ ADF UNIT ROOT TEST BY STATIONS } \\
\hline \% rejections with all stations & \multicolumn{2}{c}{89.83} \\
\% rejections with NH stations & 90.95 \\
\hline \multicolumn{3}{c}{ PANEL UNIT ROOTS TESTS } \\
\hline Levin, Lin and Chu & -45.18 & 0.000 \\
Breitung & -8.77 & 0.000 \\
Im, Pesaran and Shin & -36.78 & 0.000 \\
Fisher (ADF) & 939.88 & 0.000 \\
Fisher (PP) & 1024.64 & 0.000 \\
\hline \hline
\end{tabular}

Notes: At the top panel, percentage of rejections of the ADF test station by station, considering all stations from 1880 that have at least 30 observations. At the bottom, panel unit root tests of the 19 distributional characteristics. We use the test of Breitung (2000) and Levin et al. (2002) that assume common persistence parameters across crosssections. Fisher-type tests proposed by Maddala and Wu (1999) and Choi (2001) and those suggested by Im et al. (2003) allow different persistence parameters across cross-sections. 
Table 6

Trend test (CRU station data)

\begin{tabular}{lcc}
\hline \hline Characteristic & Coeff & p-value \\
\hline mean & 0.0102 & 0.0000 \\
max & 0.0053 & 0.0003 \\
min & 0.0188 & 0.0000 \\
std & -0.0030 & 0.0000 \\
iqr & -0.0024 & 0.0091 \\
rank & -0.0134 & 0.0004 \\
kur & 0.0012 & 0.0206 \\
skw & -0.0000 & 0.4935 \\
q5 & 0.0146 & 0.0000 \\
q10 & 0.0135 & 0.0000 \\
q20 & 0.0133 & 0.0000 \\
q30 & 0.0113 & 0.0000 \\
q40 & 0.0110 & 0.0000 \\
q50 & 0.0110 & 0.0000 \\
q60 & 0.0107 & 0.0000 \\
q70 & 0.0109 & 0.0000 \\
q80 & 0.0089 & 0.0000 \\
q90 & 0.0012 & 0.2494 \\
q95 & 0.0008 & 0.4386 \\
\hline \hline
\end{tabular}

Notes: Annual distributional characteristics of temperature with CRU station data (1880-2015). OLS estimates and HAC $t_{\beta=0}$ from regression: $C_{t}=\alpha+\beta t+u_{t}$. 
Table 7

Co-trending analysis

\begin{tabular}{|c|c|c|}
\hline Characteristic & Wald test & $\mathrm{p}$-value \\
\hline \multicolumn{3}{|l|}{ QUANTILES } \\
\hline All quantiles (q5, q10,..,q90,q95) & 42.979 & 0.000 \\
\hline Lower quantiles (q5-q30) & 2.686 & 0.443 \\
\hline Medium quantiles (q40-q60) & 0.027 & 0.987 \\
\hline Upper quantiles (q70-q95) & 21.892 & 0.000 \\
\hline \multicolumn{3}{|l|}{ SPACING } \\
\hline & Trend-coeff. & p-value \\
\hline q50-q5 & -0.004 & 0.008 \\
\hline q95-q50 & -0.010 & 0.041 \\
\hline
\end{tabular}

Notes: Annual distributional characteristics (quantiles) of temperature of CRU station data (1880-2015). At the top panel, Wald test of the null hypothesis of equality of trend coefficients of a given set of characteristics. P-values calculated from bootstrap critical values. At the bottom, the $T T$ is applied to the difference between two representative quantiles. 


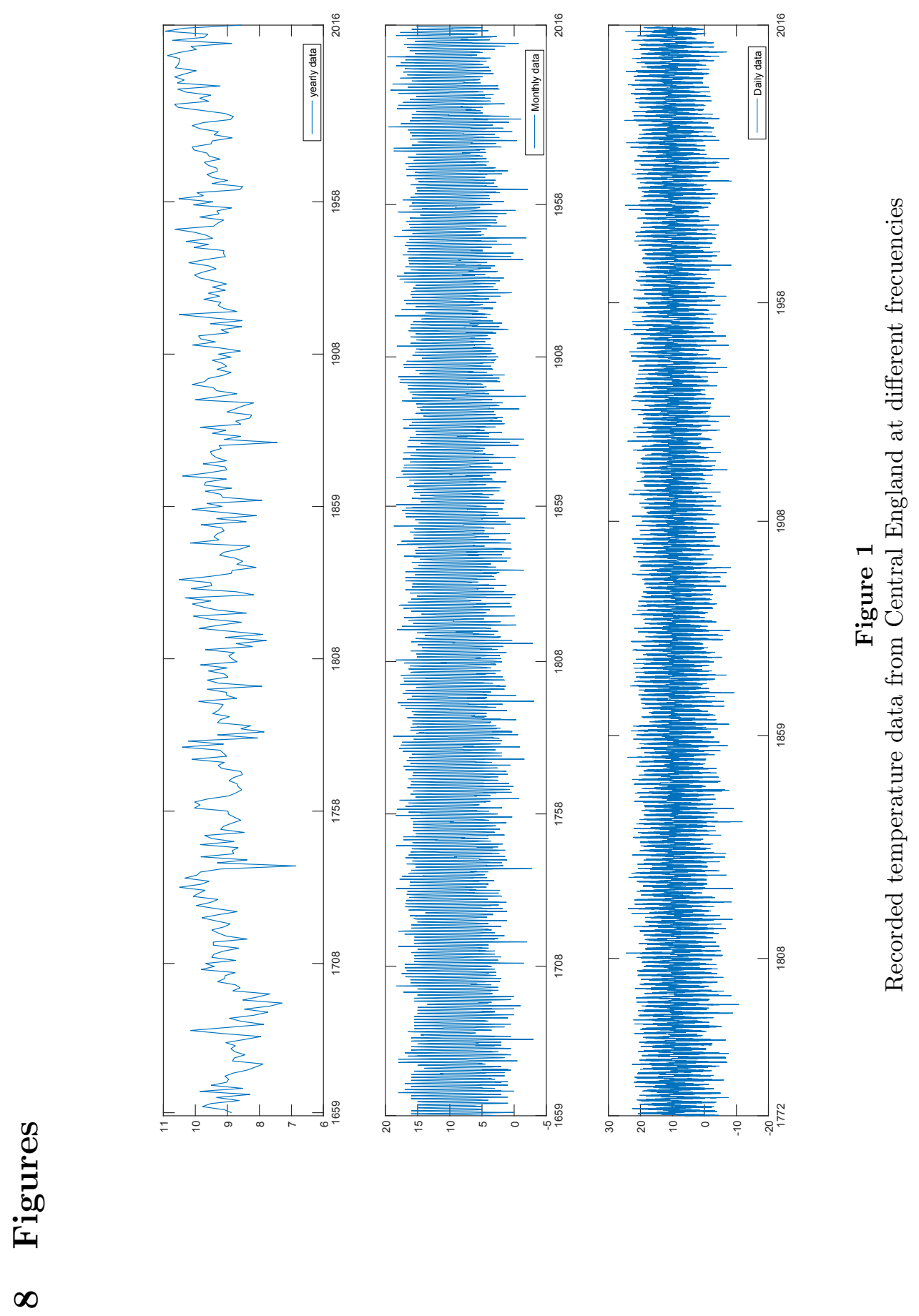




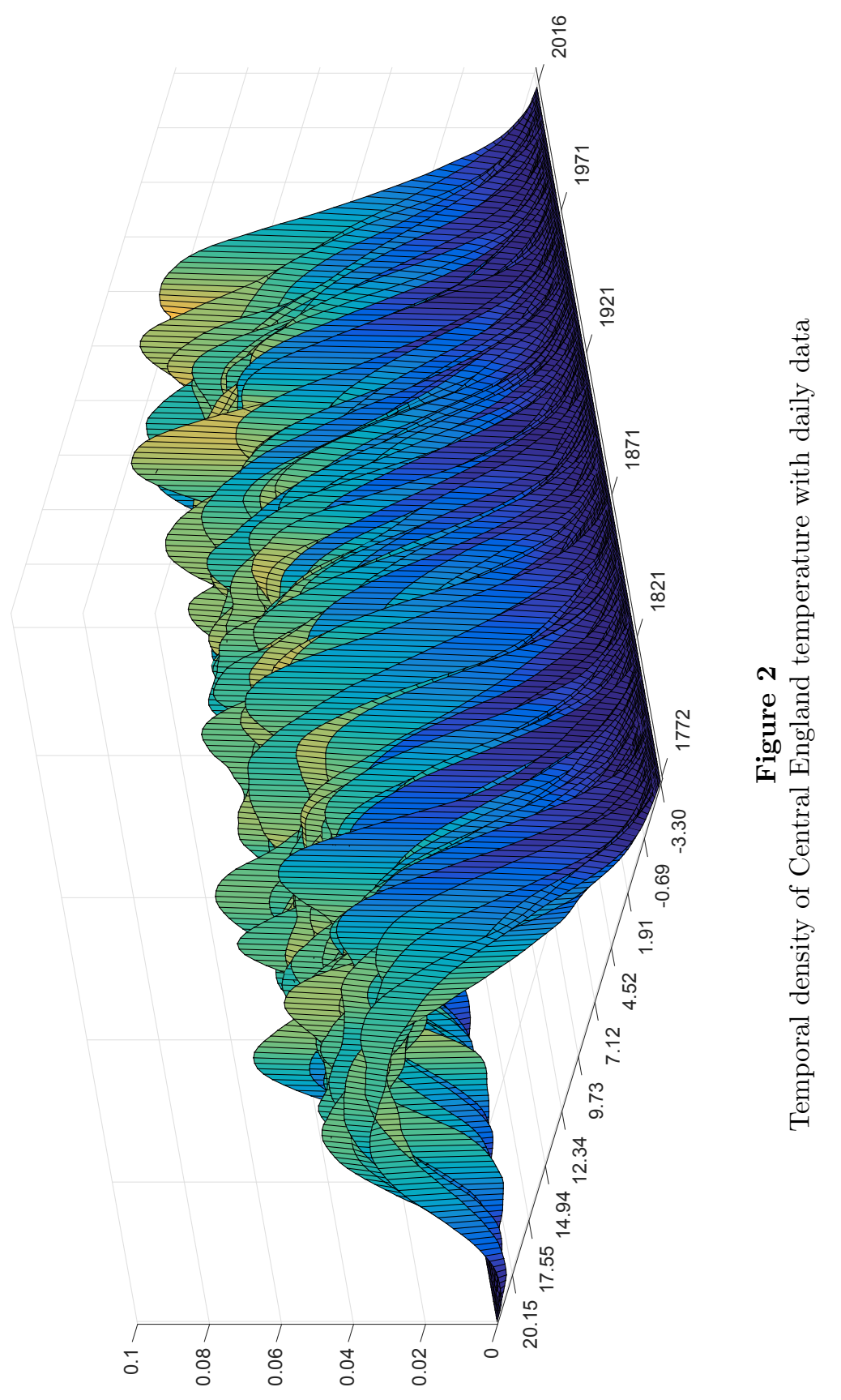




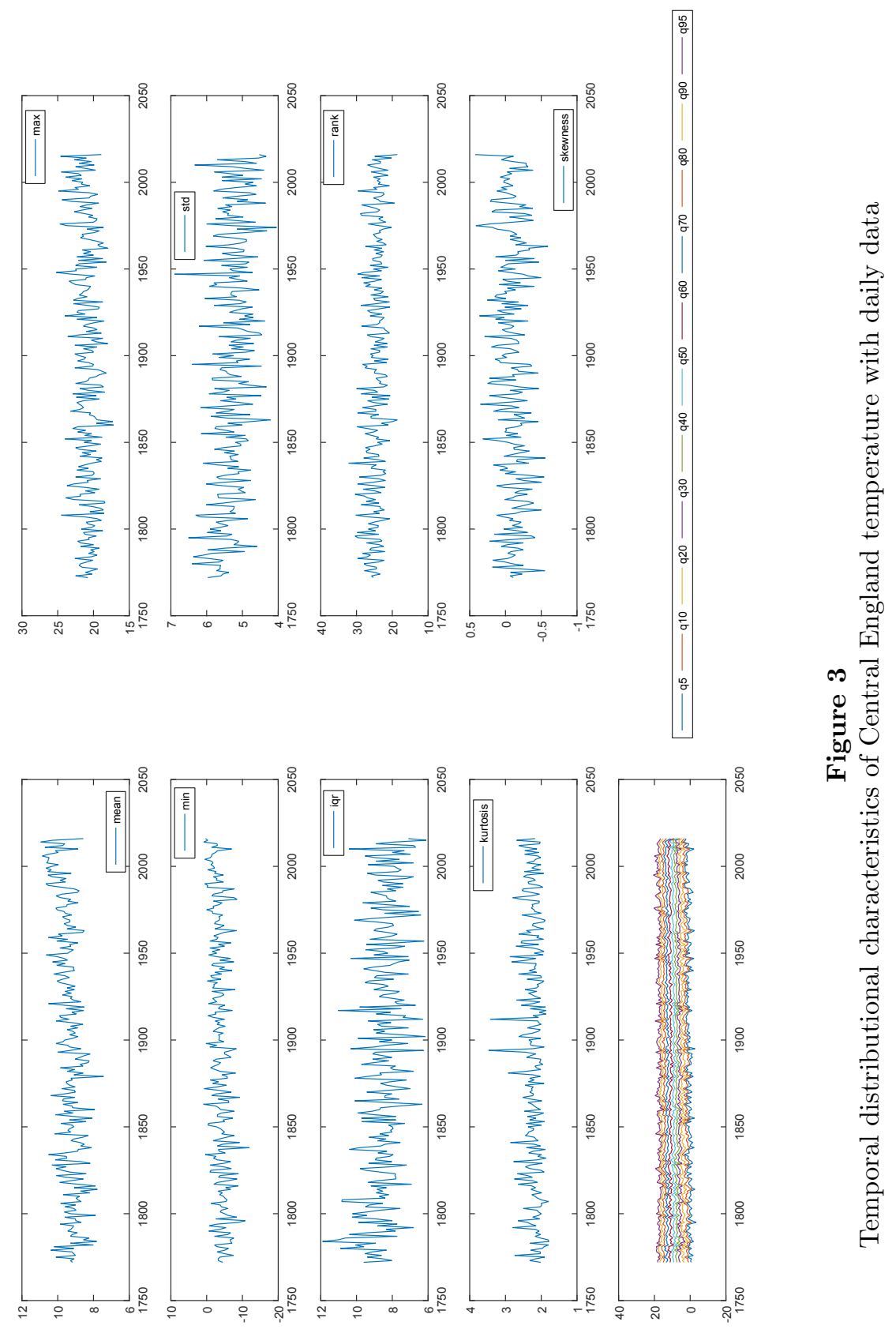




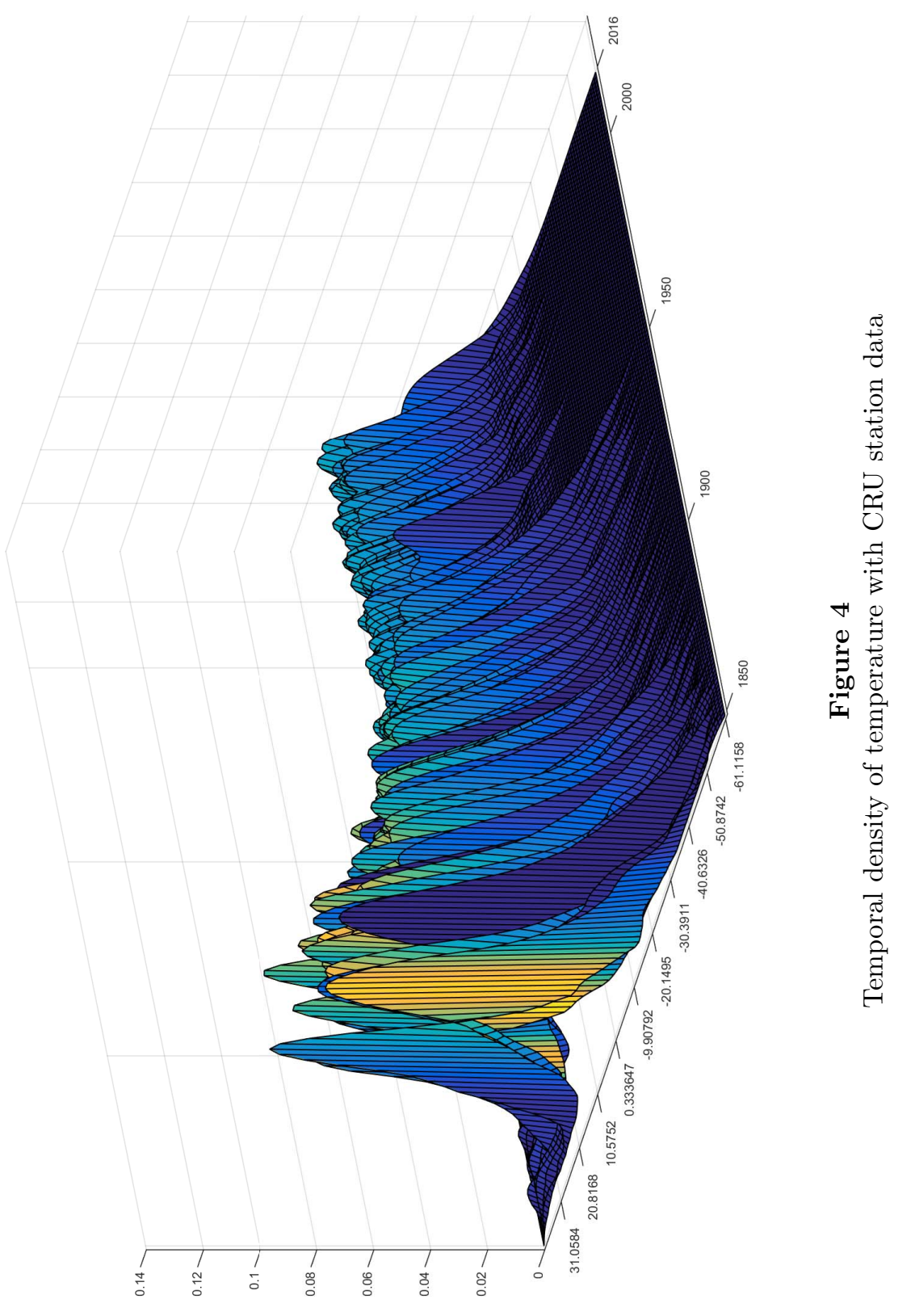




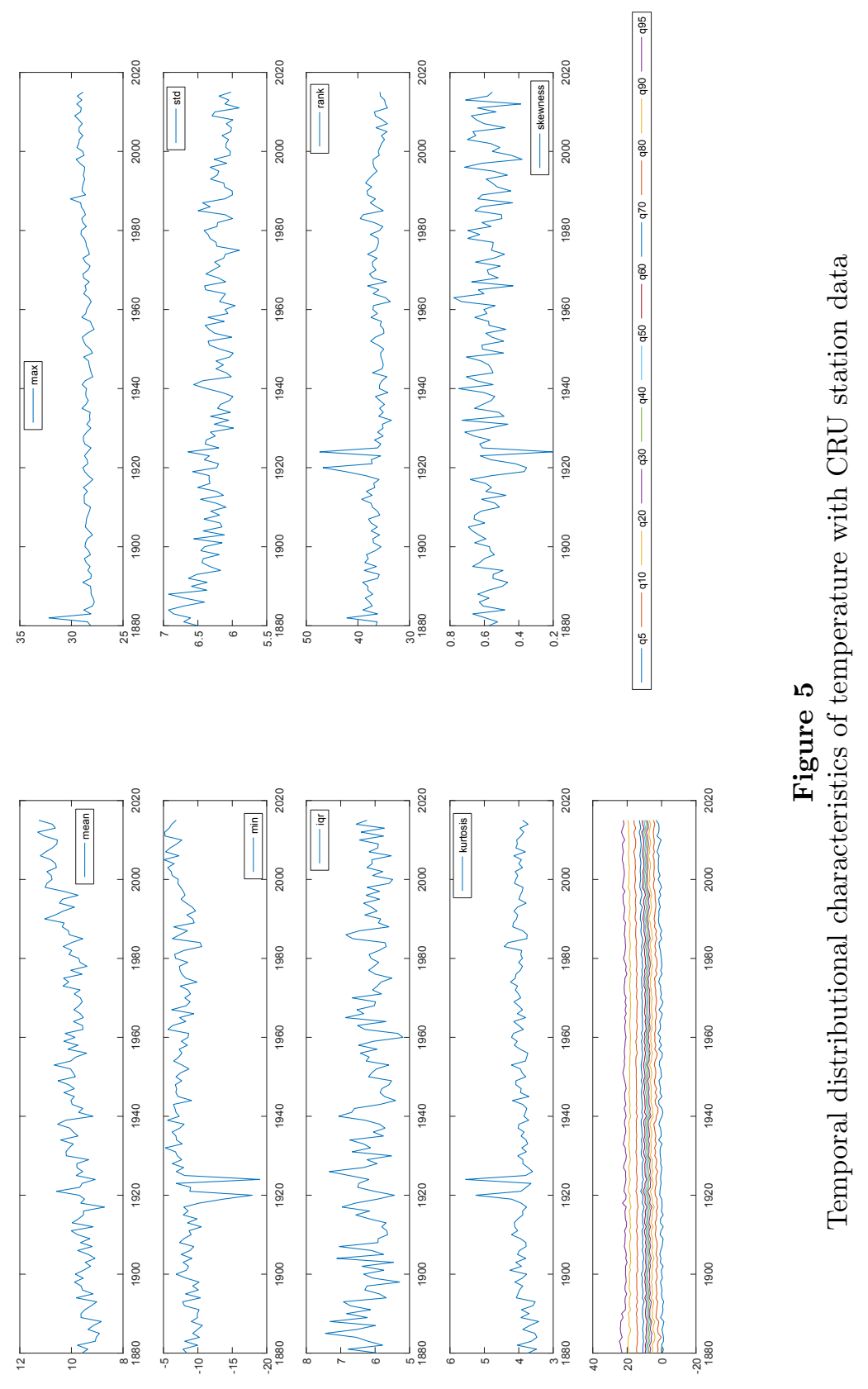




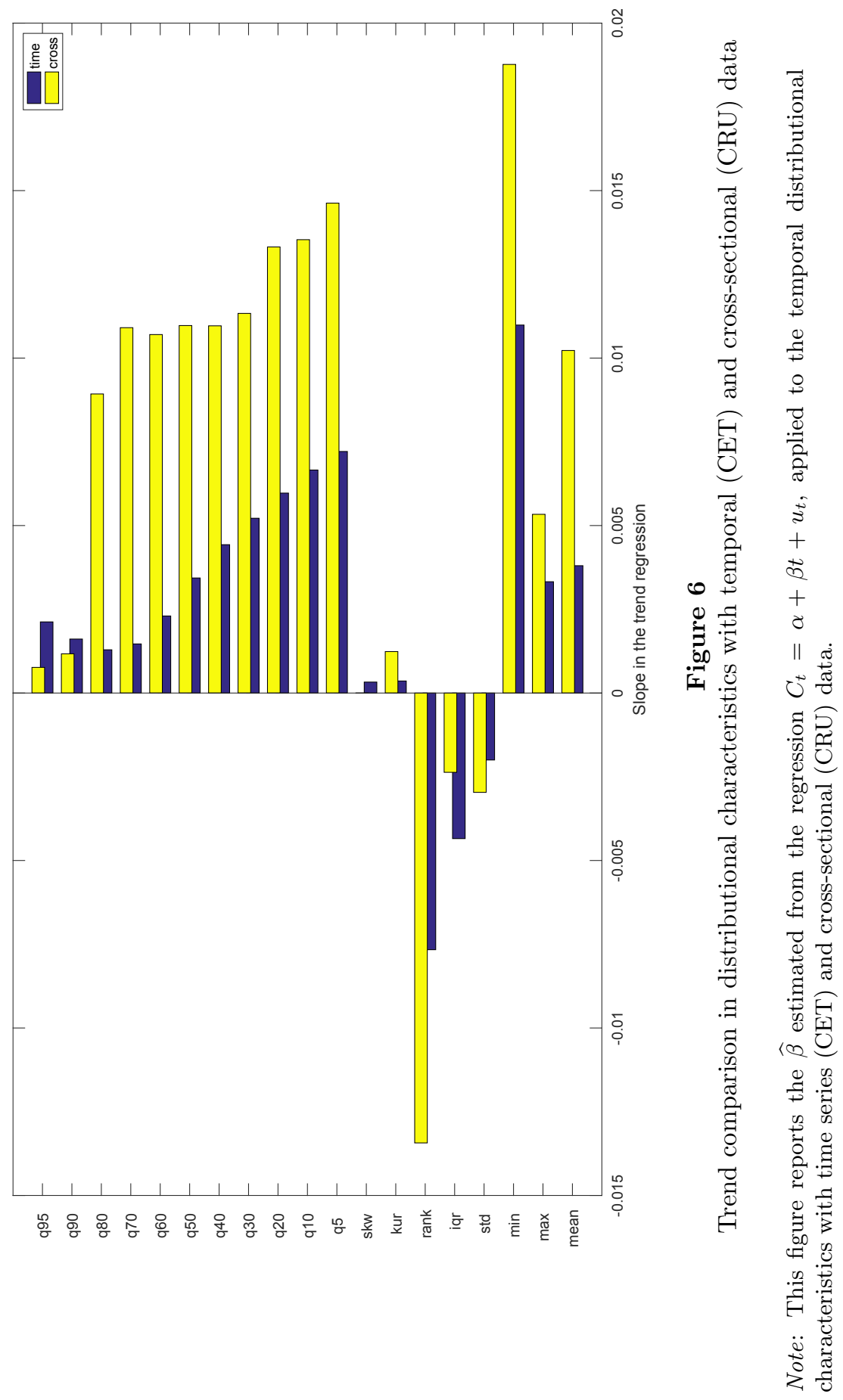




\section{Appendix}

\section{Appendix A: Proofs}

\section{Proof of Proposition 1}

See sections 16.1 and 16.2 in Hamilton (1994).

\section{Proof of Proposition 2}

Our definition of an $\mathrm{I}(0)$ process follows Johansen (1995): $I(0)=\Psi(L) \varepsilon_{t}$, with $\sum_{i=1}^{\infty} \Psi_{i} \neq 0, \sum_{j=1}^{\infty} \Psi_{j}^{2}<\infty$ and $\varepsilon_{t} \sim \operatorname{iid}\left(0, \sigma^{2}\right)$.

Part 1: Asymptotic behavior of OLS $\widehat{\beta}$ :

$$
\widehat{\beta}=\frac{\sum_{t=1}^{T}\left(C_{t}-\bar{C}\right)(t-\bar{t})}{\sum_{t=1}^{T}(t-\bar{t})^{2}}=\frac{\sum_{t=1}^{T} C_{t} t-\bar{C} \sum_{t=1}^{T} t-\bar{t} \sum_{t=1}^{T} C_{t}+\bar{C} \bar{t}}{\sum_{t=1}^{T}(t-\bar{t})^{2}}
$$

Taking into account that

$$
\begin{aligned}
& \sum_{t=1}^{T} C_{t} t=O_{p}\left(T^{2+\delta}\right), \\
& \bar{C} \sum_{t=1}^{T} t=O_{p}\left(T^{2+\delta}\right), \\
& \bar{t} \sum_{t=1}^{T} C_{t}=O_{p}\left(T^{2+\delta}\right), \\
& \bar{C} \bar{t}=O_{p}\left(T^{1+\delta}\right)
\end{aligned}
$$

and

$$
\sum_{t=1}^{T}(t-\bar{t})^{2}=O\left(T^{3}\right)
$$

we obtain that $\widehat{\beta}=O_{p}\left(T^{\delta-1}\right)$.

Part 2: Asymptotic behaviour of $t_{\beta=0}$ :

$$
t_{\beta=0}=\frac{\widehat{\beta}-0}{\sqrt{\widehat{\sigma}_{u}^{2} / \sum_{t=1}^{T}(t-\bar{t})^{2}}}=\frac{\sum_{t=1}^{T}\left(C_{t}-\bar{C}\right)(t-\bar{t})}{\sqrt{\widehat{\sigma}_{u}^{2} \sum_{t=1}^{T}(t-\bar{t})^{2}}}
$$


From Part 1 the numerator is $O_{p}\left(T^{2+\delta}\right)$. It is easy to obtain that

$$
\widehat{\sigma}_{u}^{2}=\frac{\sum_{t=1}^{T}\left(C_{t}-\widehat{\alpha}-\widehat{\beta} t\right)^{2}}{T}=\left\{\begin{array}{l}
O_{p}\left(T^{(1+2 \delta-\gamma}\right) \text { for } 0 \leq \gamma \leq 1 \\
O_{p}\left(T^{2 \delta}\right) \text { for } 1 \leq \gamma \leq 1+\delta
\end{array}\right.
$$

Taking into account that $\left.\sum_{t=1}^{T}(t-\bar{t})^{2}\right)=O\left(T^{3}\right)$, the result follows.

\section{Proof of Proposition 3}

For the fractional case, $1 / 2<d<3 / 2$, see Marmol and Velasco (2002).

For the near unit root as well as for the local level model, the proof follows straightforward from the proof in Durlauf and Phillips (1988) for the pure I(1) case.

\section{Appendix B: Finite-sample performance}

In this appendix the finite-sample performance of our proposed trend test $(T T)$ is analyzed via Monte Carlo experiment. Sample sizes are T $=200,500$, and 1000. Number of replications equal to 10,000. In all cases, the significance level is $5 \%$ (critical values for a $\mathrm{N}(0,1)$ ) and a $\mathrm{HAC} t_{\beta=0}$ is used. In general the parameters of a given model have been estimated or selected by fitting that model to the average annual Central England temperature (1772-2015). However, in some cases (superexponential trends, Gompertz curves and logistic trends) when the fitting is very unstable we use other typical economic series such as the UK nominal GDP percapita (1800-2010) (from Madisson, 2013) and others (Population, IPI and Wholesale Prices) from Davis (1941).

\section{SIZE}

The empirical size is investigated by generating several non-trending models.

- Case 1: A white noise model (WN) from a Normal $(0,1)$.

- Case 2: $\sin (u * t), t=1, \ldots, T, \mathrm{u} \sim U(0,1)$, where $u$ is used to reduce the frequency of the sin function.

- Case 3: An $\operatorname{AR}(2)$ process which parameter values obtained from fitting an $\operatorname{AR}(2)$ to the average annual Central England temperature. 
- Case 4: $\operatorname{An} \operatorname{AR}(2)$ with complex roots. The first parameter is selected from a $U(0,1)$ and the second one from a $U(-1,0)$

Table 8

Size of $T T$

\begin{tabular}{lccc}
\hline \hline & $\mathrm{T}=200$ & $\mathrm{~T}=500$ & $\mathrm{~T}=1000$ \\
\hline $\mathrm{WN}$ & 0.0693 & 0.0583 & 0.0579 \\
$\sin \left(\mathrm{u}^{*} \mathrm{t}\right), \mathrm{u} \sim U(0,1)$ & 0.0582 & 0.0377 & 0.0388 \\
$\mathrm{AR}(2)$ with estimated parameters & 0.1391 & 0.1150 & 0.0994 \\
complex roots & 0.0499 & 0.0445 & 0.0389 \\
\hline \hline
\end{tabular}

\section{POWER}

Deterministic trends (See Proposition 2): The power of our TT is investigated by generating data from the main deterministic trends used in the literature plus a $\mathrm{N}(0,1)$ white noise term:

(I) Polynomial Trends

$$
x(t)=a_{0}+a_{1} t+a_{2} t^{2}+\ldots .+a_{p} t^{k}
$$

with $k=1, k=2, k$ chosen by a SBIC. We also analyze the case of $k=\theta$ with $\theta<1$. In all these cases the parameters have been estimated or selected by fitting the corresponding polynomial trend to the average annual Central England temperature. (II) Exponential trends

$$
x(t)=a_{0}+a_{1} e^{\lambda t}
$$

- Sub-exponential:

$$
x(t)=a_{0}+a_{1} e^{a_{2} t^{\lambda}}
$$

with $\lambda<1$.

- Super-exponential:

$$
x(t)=a_{0}+a_{1} e^{e^{\lambda t}}
$$

The Gompertz curve can be included within this sub-case:

$$
x(t)=e^{a_{0}-a_{1} e^{-\lambda t}}
$$


(III) Logistic Trends

$$
x(t)=\frac{a_{1}}{1+a_{2} e^{-\lambda t}}
$$

(IV) Segmented Trends

$$
x(t)=a_{0}+b_{0} d_{1 t}+a_{1} t+b_{2} d_{1 t} t
$$

with $d_{1 t}$ being a dummy variable that takes the value 1 in regime $\mathrm{A}$ and 0 in regime $\mathrm{B}$.

(V) Logistic Smooth Transition Trends

$$
x(t)=a_{0}+a_{1} t+\left(b_{0}+b_{2} t\right) S_{t}(\theta, \tau)
$$

with $S_{t}(\theta, \tau)=(1+\exp (-\theta(t-\tau T)))^{-1}$.

\section{Table 9}

Power (deterministic trends) of $T T$

\begin{tabular}{lccc}
\hline \hline & $\mathrm{T}=200$ & $\mathrm{~T}=500$ & $\mathrm{~T}=1000$ \\
\hline Polynomial trend $\mathrm{k}=1$ & 0.9998 & 1.0000 & 1.0000 \\
Polynomial trend $\mathrm{k}=2$ & 0.9119 & 1.0000 & 1.0000 \\
Polynomial trend $\mathrm{k}=$ sbic & 0.9083 & 1.0000 & 1.0000 \\
Polynomial trend $k=\theta<1$ & 0.9937 & 1.0000 & 1.0000 \\
Exponential & 0.8782 & 1.0000 & 1.0000 \\
Exponential (sub) & 0.8718 & 1.0000 & 1.0000 \\
Exponential (super, UK GDP) & 1.0000 & $-\left(^{*}\right)$ & $-\left(^{*}\right)$ \\
Exponential (Gompertz curve, UK GDP) & 1.0000 & $-\left(^{*}\right)$ & $-\left(^{*}\right)$ \\
Logistic (Population) & 1.0000 & 1.0000 & 1.0000 \\
Logistic (Industrial Production Index) & 1.0000 & 1.0000 & 1.0000 \\
Logistic (Wholesale Prices) & 1.0000 & 1.0000 & 1.0000 \\
Segmented trends & 1.0000 & 1.0000 & 1.0000 \\
Logistic smooth transition (UK GDP) & 1.0000 & 1.0000 & 1.0000 \\
\hline \hline
\end{tabular}

Stochastic trends (see Proposition 3) Following Müeller and Watson (2008) we consider the three most common long-run models generating stochastic trends: fractional models $(1 / 2<d<3 / 2)$, near unit root models and local level models. 
Table 10

Power (stochastic trends) of $T T$

\begin{tabular}{|c|c|c|c|c|c|c|}
\hline \multicolumn{7}{|c|}{ FRACTIONAL MODEL } \\
\hline$d$ & 0.5 & 0.7 & 0.9 & 1 & 1.2 & 1.5 \\
\hline $\mathrm{T}=50$ & 0.4334 & 0.5512 & 0.6600 & 0.7089 & 0.7868 & 0.8715 \\
\hline $\mathrm{T}=100$ & 0.4776 & 0.6132 & 0.7112 & 0.7562 & 0.8240 & 0.8887 \\
\hline $\mathrm{T}=200$ & 0.5326 & 0.6582 & 0.7613 & 0.8058 & 0.8642 & 0.9096 \\
\hline $\mathrm{T}=300$ & 0.5722 & 0.7103 & 0.7943 & 0.8314 & 0.8829 & 0.9285 \\
\hline $\mathrm{T}=50$ & 0.6102 & 0.7442 & 0.8253 & 0.8566 & 0.8990 & 0.9423 \\
\hline $\mathrm{T}=1000$ & 0.6712 & 0.7913 & 0.8711 & 0.8928 & 0.9253 & 0.9536 \\
\hline \multicolumn{7}{|c|}{ NEAR UNIT ROOT } \\
\hline & $c=30$ & $\mathrm{c}=10$ & $\mathrm{c}=5$ & $\mathrm{c}=0$ & & \\
\hline $\mathrm{T}=50$ & 0.1521 & 0.3537 & 0.4897 & 0.7180 & & \\
\hline $\mathrm{T}=100$ & 0.2163 & 0.4350 & 0.5572 & 0.7649 & & \\
\hline $\mathrm{T}=200$ & 0.2879 & 0.5197 & 0.6262 & 0.8060 & & \\
\hline $\mathrm{T}=300$ & 0.3633 & 0.5860 & 0.6850 & 0.8317 & & \\
\hline $\mathrm{T}=50$ & 0.4320 & 0.6387 & 0.7324 & 0.8573 & & \\
\hline $\mathrm{T}=1000$ & 0.5378 & 0.7193 & 0.7862 & 0.8989 & & \\
\hline \multicolumn{7}{|c|}{ LOCAL LEVEL MODEL } \\
\hline$q$ & 0 & 0.1 & 0.5 & 1 & 5 & 10 \\
\hline $\mathrm{T}=50$ & 0.7180 & 0.7193 & 0.7151 & 0.7079 & 0.5312 & 0.3347 \\
\hline $\mathrm{T}=100$ & 0.7649 & 0.7638 & 0.7635 & 0.7609 & 0.6807 & 0.5369 \\
\hline $\mathrm{T}=200$ & 0.8060 & 0.8058 & 0.8057 & 0.8052 & 0.7722 & 0.6967 \\
\hline $\mathrm{T}=300$ & 0.8317 & 0.8317 & 0.8312 & 0.8306 & 0.8137 & 0.7686 \\
\hline $\mathrm{T}=50$ & 0.8573 & 0.8572 & 0.8567 & 0.8573 & 0.8467 & 0.8218 \\
\hline $\mathrm{T}=1000$ & 0.8989 & 0.8987 & 0.8986 & 0.8984 & 0.8967 & 0.8862 \\
\hline
\end{tabular}




\section{Appendix C: Additional time series data of temperature}

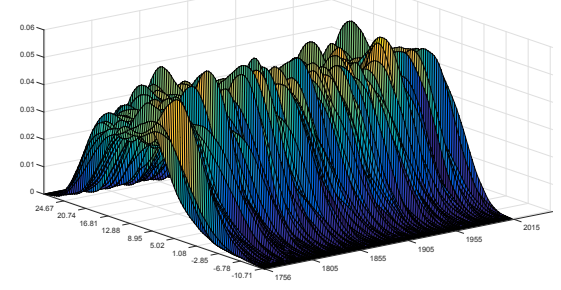

(a) Stockolm

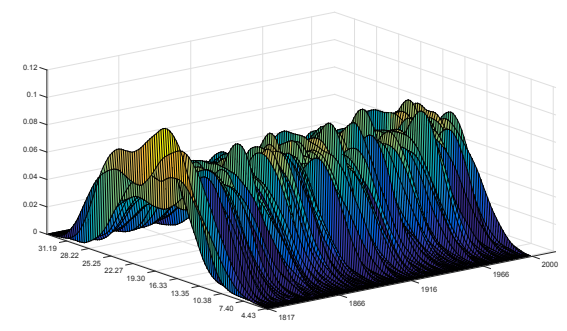

(b) Cadiz

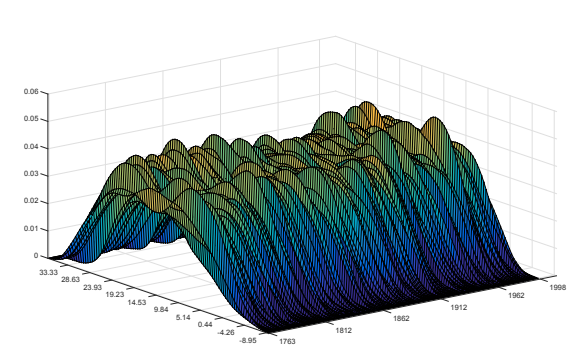

(c) Milan

Figure 7

Temporal density of temperature with daily data (IMPROVE project) 
Table 11

Unit root tests (IMPROVE data)

\begin{tabular}{lccc}
\hline \hline Characteristic & Stockholm & Cadiz & Milan \\
\hline mean & -13.34 & -5.94 & -12.77 \\
max & $(0.000)$ & $(0.000)$ & $(0.000)$ \\
& -16.96 & -8.45 & -13.20 \\
min & $(0.000)$ & $(0.000)$ & $(0.000)$ \\
& -14.86 & -8.97 & -13.88 \\
std & $(0.000)$ & $(0.000)$ & $(0.000)$ \\
& -13.99 & -8.46 & -13.94 \\
iqr & $(0.000)$ & $(0.000)$ & $(0.000)$ \\
& -14.14 & -9.65 & -15.07 \\
rank & $(0.000)$ & $(0.000)$ & $(0.000)$ \\
& -15.88 & -11.77 & -13.50 \\
kur & $(0.000)$ & $(0.000)$ & $(0.000)$ \\
& -17.07 & -14.37 & -13.81 \\
skw & $(0.000)$ & $(0.000)$ & $(0.000)$ \\
& -14.18 & -12.57 & -13.62 \\
q5 & $(0.000)$ & $(0.000)$ & $(0.000)$ \\
& -13.51 & -6.28 & -14.35 \\
q10 & $(0.000)$ & $(0.000)$ & $(0.000)$ \\
& -13.58 & -8.38 & -14.31 \\
q20 & $(0.000)$ & $(0.000)$ & $(0.000)$ \\
& -13.93 & -8.13 & -13.71 \\
q30 & $(0.000)$ & $(0.000)$ & $(0.000)$ \\
& -13.82 & -7.79 & -14.55 \\
q40 & $(0.000)$ & $(0.000)$ & $(0.000)$ \\
q95 & -13.57 & -6.76 & -13.85 \\
q50 & $(0.000)$ & $(0.000)$ & $(0.000)$ \\
& -13.37 & -6.91 & -13.69 \\
q70 & $(0.000)$ & $(0.000)$ & $(0.000)$ \\
& -3.26 & -10.61 & -8.87 \\
& $(0.076)$ & $(0.000)$ & $(0.000)$ \\
& -13.41 & -5.82 & -13.03 \\
& $(0.000)$ & $(0.000)$ & $(0.000)$ \\
& -13.66 & -3.41 & -5.77 \\
& $(0.000)$ & $(0.053)$ & $(0.000)$ \\
& -14.91 & -4.11 & -6.18 \\
& $(0.000)$ & $(0.008)$ & $(0.000)$ \\
& -15.85 & -12.06 & -13.33 \\
& $(0.000)$ & $(0.000)$ & $(0.000)$ \\
\hline & & & \\
& & & \\
& & &
\end{tabular}

Notes: Annual distributional characteristics of temperature from IMPROVE daily data. Data from Stockholm (1756-2012), Cadiz (1817-2000) and Milan (1763-1998) from Camuffo and Jones (2002). Stockholm temperatures are been update to 2015 by Bolin Center Database. P-values in brackets. Lag-selection according to SBIC criterion. 
Table 12

Trend test (IMPROVE data, Stockholm)

\begin{tabular}{lcc}
\hline \hline Characteristic & Coeff & p-value \\
\hline mean & 0.0042 & 0.0001 \\
max & 0.0012 & 0.2198 \\
min & 0.0221 & 0.0000 \\
std & -0.0038 & 0.0000 \\
iqr & -0.0045 & 0.0002 \\
rank & -0.0210 & 0.0000 \\
kur & -0.0006 & 0.0041 \\
skw & 0.0005 & 0.0094 \\
q5 & 0.0126 & 0.0000 \\
q10 & 0.0099 & 0.0000 \\
q20 & 0.0060 & 0.0001 \\
q30 & 0.0046 & 0.0000 \\
q40 & 0.0050 & 0.0001 \\
q50 & 0.0045 & 0.0005 \\
q60 & 0.0031 & 0.0116 \\
q70 & 0.0016 & 0.0858 \\
q80 & 0.0000 & 0.4865 \\
q90 & -0.0006 & 0.3398 \\
q95 & -0.0001 & 0.4660 \\
\hline \hline
\end{tabular}

Notes: Annual distributional characteristics of temperature from daily Stockholm data (1756-2012). OLS estimates and HAC $t_{\beta=0}$ from regression: $C_{t}=$ $\alpha+\beta t+u_{t}$. 
Table 13

Trend test (IMPROVE data, Cadiz)

\begin{tabular}{lcc}
\hline \hline Characteristic & Coeff & p-value \\
\hline mean & 0.0047 & 0.0000 \\
max & 0.0070 & 0.0042 \\
min & 0.0070 & 0.0489 \\
std & -0.0006 & 0.2648 \\
iqr & -0.0000 & 0.4999 \\
rank & -0.0037 & 0.0119 \\
kur & 0.0009 & 0.0011 \\
skw & 0.0002 & 0.2565 \\
q5 & 0.0050 & 0.0132 \\
q10 & 0.0057 & 0.0009 \\
q20 & 0.0059 & 0.0001 \\
q30 & 0.0060 & 0.0001 \\
q40 & 0.0053 & 0.0009 \\
q50 & 0.0042 & 0.0020 \\
q60 & 0.0044 & 0.0007 \\
q70 & 0.0040 & 0.0027 \\
q80 & 0.0018 & 0.0798 \\
q90 & 0.0036 & 0.0065 \\
q95 & 0.0059 & 0.0002 \\
\hline \hline
\end{tabular}

Notes: Annual distributional characteristics of temperature from daily Cadiz data (1812-2000). OLS estimates and HAC $t_{\beta=0}$ from regression: $C_{t}=\alpha+\beta t+u_{t}$. 
Table 14

Trend test (IMPROVE data, Milan)

\begin{tabular}{lcc}
\hline \hline Characteristic & Coeff & p-value \\
\hline mean & 0.0027 & 0.0002 \\
max & 0.0023 & 0.0679 \\
min & 0.0120 & 0.0000 \\
std & -0.0025 & 0.0000 \\
iqr & -0.0058 & 0.0000 \\
rank & -0.0097 & 0.0001 \\
kur & 0.0002 & 0.0492 \\
skw & 0.0004 & 0.0015 \\
q5 & 0.0067 & 0.0000 \\
q10 & 0.0068 & 0.0000 \\
q20 & 0.0061 & 0.0000 \\
q30 & 0.0047 & 0.0000 \\
q40 & 0.0033 & 0.0034 \\
q50 & 0.0006 & 0.3130 \\
q60 & -0.0008 & 0.2091 \\
q70 & -0.0005 & 0.3253 \\
q80 & -0.0001 & 0.4591 \\
q90 & 0.0007 & 0.2802 \\
q95 & 0.0021 & 0.0437 \\
\hline \hline
\end{tabular}

Notes: Annual distributional characteristics of temperature from daily Milan data (1763-1998). OLS estimates and HAC $t_{\beta=0}$ from regression: $C_{t}=\alpha+\beta t+u_{t}$. 


\section{Appendix D: Additional cross-sectional data of temperature (Grids instead of raw stations}

)

Table 15

$\mathrm{ADF}$ unit root test (CRU grid data)

\begin{tabular}{lccc}
\hline \hline Characteristic & ADF-SBIC & p-value & lags \\
\hline mean & -4.42 & 0.004 & 1 \\
max & -5.61 & 0.000 & 1 \\
min & -9.87 & 0.000 & 0 \\
std & -3.55 & 0.039 & 3 \\
iqr & -5.59 & 0.000 & 1 \\
rank & -11.05 & 0.000 & 0 \\
kur & -10.32 & 0.000 & 0 \\
skw & -9.64 & 0.000 & 0 \\
q5 & -9.50 & 0.000 & 0 \\
q10 & -8.86 & 0.000 & 0 \\
q20 & -5.08 & 0.000 & 1 \\
q30 & -4.62 & 0.002 & 1 \\
q40 & -4.40 & 0.004 & 1 \\
q50 & -4.26 & 0.005 & 1 \\
q60 & -4.12 & 0.008 & 1 \\
q70 & -3.97 & 0.012 & 1 \\
q80 & -3.97 & 0.012 & 1 \\
q90 & -4.30 & 0.005 & 1 \\
q95 & -7.36 & 0.000 & 0 \\
\hline Global CRU, yearly & -1.28 & 0.887 & 3 \\
Global CRU, montly & -20.28 & 0.000 & 0 \\
Northern CRU, yearly & -1.05 & 0.932 & 3 \\
Northern CRU, monthly & -22.21 & 0.000 & 0 \\
Southern CRU, yearly & -7.16 & 0.000 & 0 \\
Southern CRU, monthly & -22.70 & 0.000 & 0 \\
\hline \hline
\end{tabular}

Notes: Annual distributional characteristics from CRU grid data (1880-2015). Lag-selection according to SBIC. criterion. 
Table 16

Additional unit root analysis (CRU grid data)

\begin{tabular}{lcc}
\hline \hline \multicolumn{2}{l}{ UnIT RoOT TESTS BY GRIDS (ADF-SBIC) } \\
\hline \multicolumn{3}{c}{83.61} \\
\hline \multicolumn{3}{c}{ PANEL rections UNIT ROOTS TESTS } \\
\hline Levin, Lin and Chu & -32.47 & 0.000 \\
Breitung & -5.14 & 0.000 \\
Im, Pesaran and Shin & -26.94 & 0.000 \\
Fisher (ADF) & 654.62 & 0.000 \\
Fisher (PP) & 1002.48 & 0.000 \\
\hline \hline
\end{tabular}

Notes: At the top of the Table we present the percentage of rejections of the ADF grid by grid, considering all grids from 1850 that have at least 30 observations. At the bottom, panel unit root tests of the 19 distributional characteristics. We use the test of Breitung (2000) and Levin et al. (2002) that assume common persistence parameters across cross-sections. Fisher-type tests proposed by Maddala and Wu (1999) and Choi (2001) and those suggested by Im et al. (2003) allow different persistence parameters across cross-sections. 
Table 17

Trend test (grid data 1880-2015)

\begin{tabular}{lcc}
\hline \hline Characteristic & Coeff & p-value \\
\hline mean & 0.0096 & 0.0000 \\
max & 0.0092 & 0.0000 \\
min & 0.0161 & 0.0000 \\
std & -0.0010 & 0.0126 \\
iqr & -0.0008 & 0.1097 \\
rank & -0.0070 & 0.0073 \\
kur & -0.0023 & 0.2546 \\
skw & 0.0046 & 0.0004 \\
q5 & 0.0125 & 0.0000 \\
q10 & 0.0112 & 0.0000 \\
q20 & 0.0101 & 0.0000 \\
q30 & 0.0096 & 0.0000 \\
q40 & 0.0093 & 0.0000 \\
q50 & 0.0090 & 0.0000 \\
q60 & 0.0089 & 0.0000 \\
q70 & 0.0089 & 0.0000 \\
q80 & 0.0090 & 0.0000 \\
q90 & 0.0090 & 0.0000 \\
q95 & 0.0087 & 0.0000 \\
\hline \hline
\end{tabular}

Notes: Annual distributional characteristics of temperature from CRU grid data (1880-2015). OLS estimates and HAC $t_{\beta=0}$ from regression: $C_{t}=\alpha+\beta t+u_{t}$. 
Table 18

Co-trending analysis

\begin{tabular}{|c|c|c|}
\hline Characteristic & Wald test & p-value \\
\hline \multicolumn{3}{|c|}{ QUANTILES } \\
\hline All quantiles (q5, q10,..,q90,q95) & 8.838 & 0.548 \\
\hline Lower quantiles (q5-q30) & 3.792 & 0.285 \\
\hline Medium quantiles (q40-q60) & 0.064 & 0.968 \\
\hline Upper quantiles (q70-q95) & 0.023 & 0.999 \\
\hline \multicolumn{3}{|l|}{ SPACING } \\
\hline & Trend-coeff. & t-pvalue \\
\hline q50-q5 & -0.003 & 0.000 \\
\hline q95-q50 & -0.000 & 0.704 \\
\hline
\end{tabular}

Notes: Annual distributional characteristics (quantiles) of temperature from CRU grid data (1880-2015). At the top panel, Wald test of the null hypothesis of equality of trend coefficients of a given set of characteristics. P-values calculated from bootstrap critical values. At the bottom, the $T T$ is applied to the difference between two representative quantiles. 

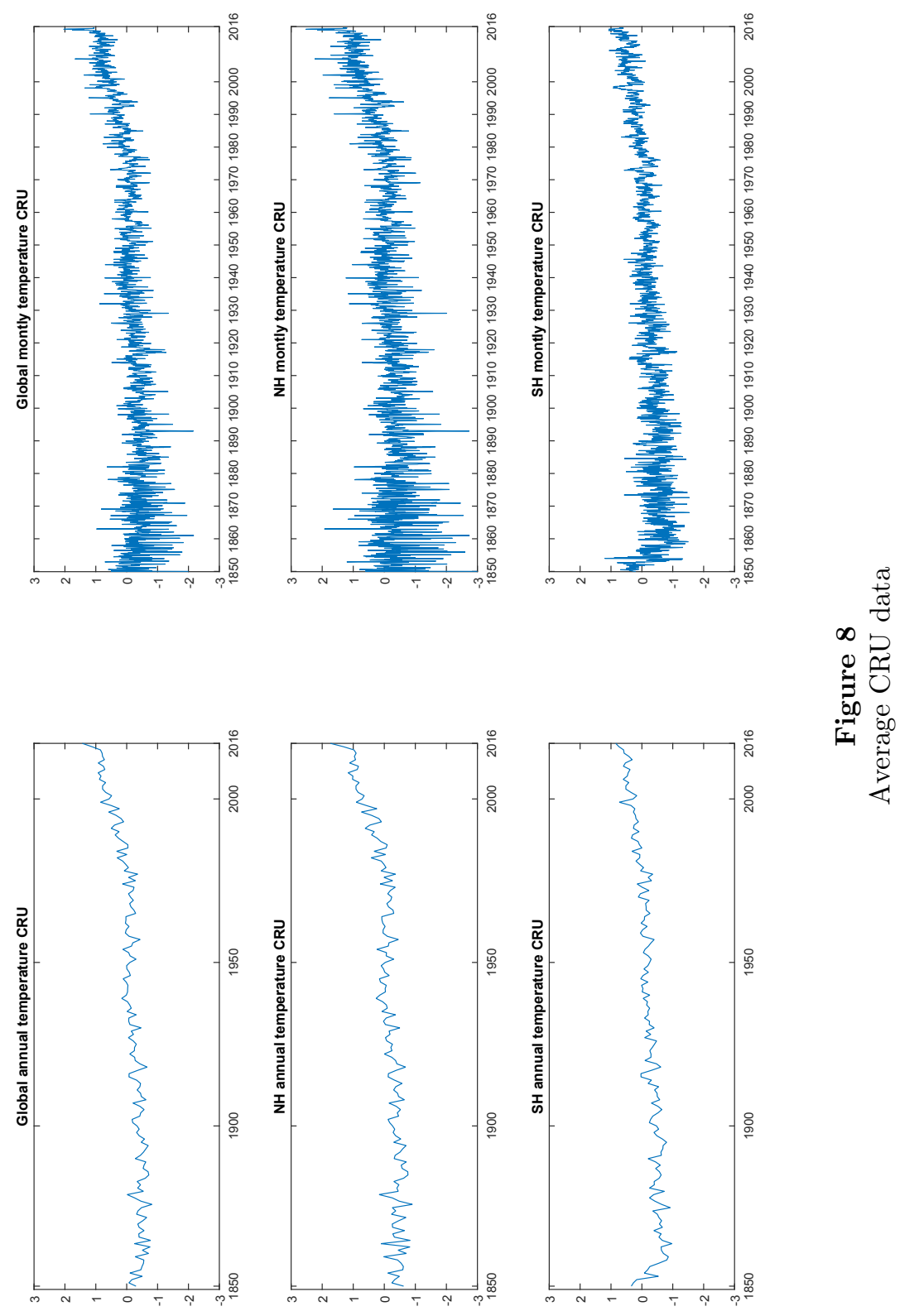


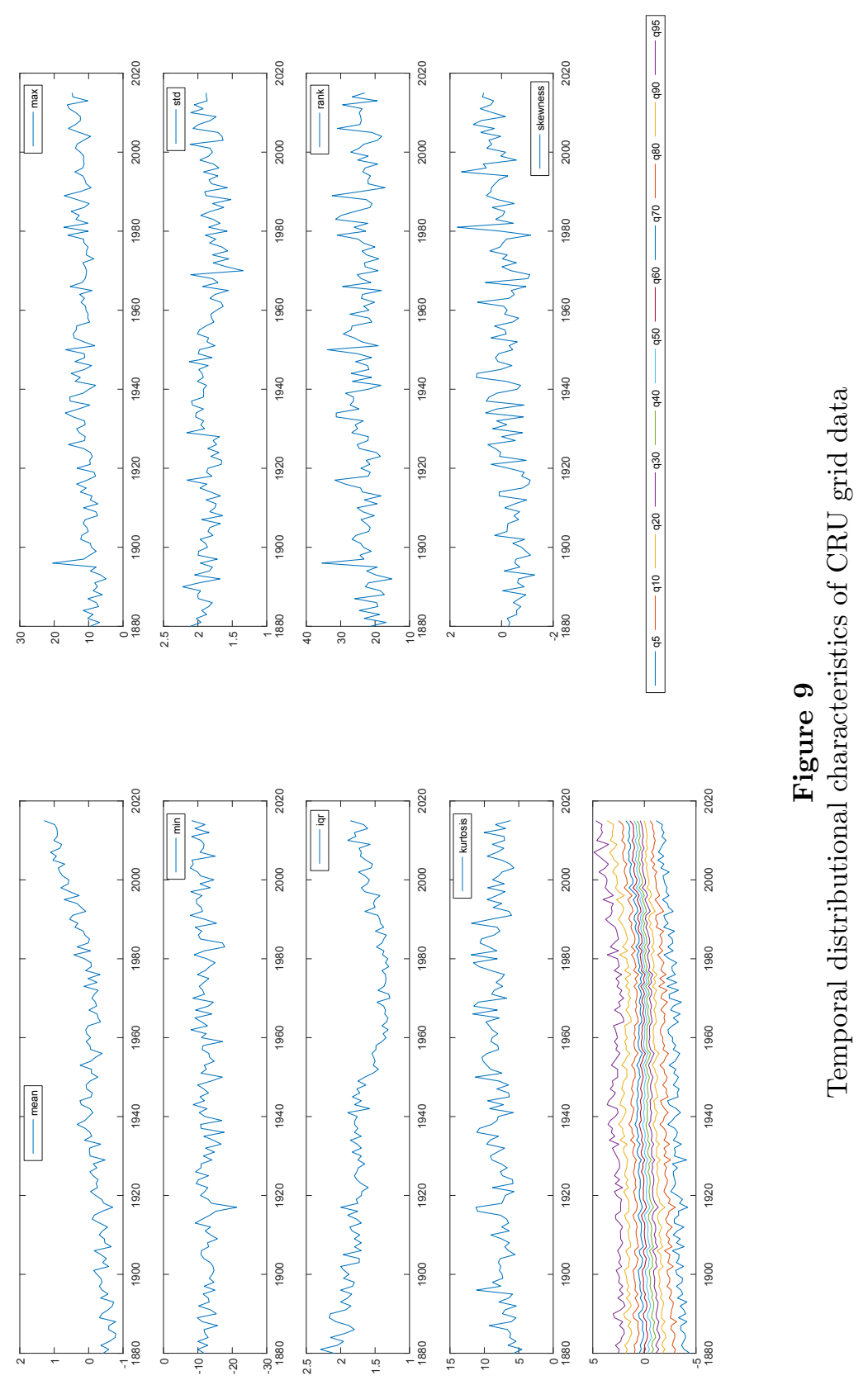

